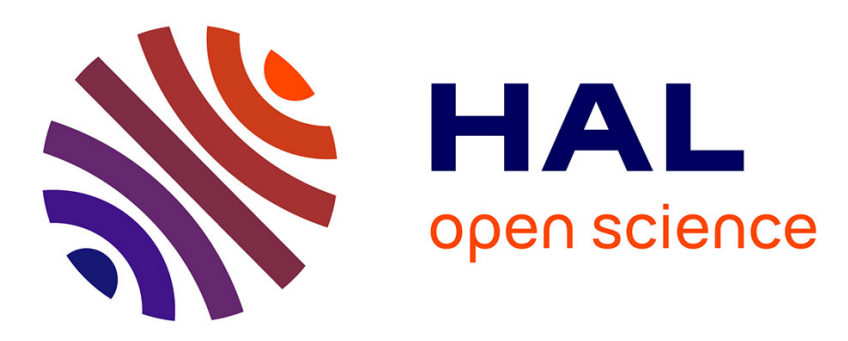

\title{
Asymptotic modeling of thermal binary monatomic gas flows in plane microchannels-Comparison with DSMC simulations
}

\author{
Renée Gatignol, Cédric Croizet
}

\section{- To cite this version:}

Renée Gatignol, Cédric Croizet. Asymptotic modeling of thermal binary monatomic gas flows in plane microchannels-Comparison with DSMC simulations. Physics of Fluids, 2017, 29 (4), pp.042001. 10.1063/1.4979683 . hal-01533304

\section{HAL Id: hal-01533304 https: / hal.sorbonne-universite.fr/hal-01533304}

Submitted on 6 Jun 2017

HAL is a multi-disciplinary open access archive for the deposit and dissemination of scientific research documents, whether they are published or not. The documents may come from teaching and research institutions in France or abroad, or from public or private research centers.
L'archive ouverte pluridisciplinaire HAL, est destinée au dépôt et à la diffusion de documents scientifiques de niveau recherche, publiés ou non, émanant des établissements d'enseignement et de recherche français ou étrangers, des laboratoires publics ou privés. 


\section{Asymptotic modeling of thermal binary monatomic gas flows in plane microchannels-Comparison with DSMC simulations}

Renée Gatignol and Cédric Croizet

Citation: Physics of Fluids 29, 042001 (2017); doi: 10.1063/1.4979683

View online: http://dx.doi.org/10.1063/1.4979683

View Table of Contents: http://aip.scitation.org/toc/phf/29/4

Published by the American Institute of Physics

\section{Articles you may be interested in}

Rarefied gas flow in converging microchannel in slip and early transition regimes

Physics of Fluids 29, 032002 (2017); 10.1063/1.4978057

Numerical investigations on aerodynamic forces of deformable foils in hovering motions

Physics of Fluids 29, 041902 (2017); 10.1063/1.4979212

New chemical-DSMC method in numerical simulation of axisymmetric rarefied reactive flow Physics of Fluids 29, 047105 (2017); 10.1063/1.4979793

Modified kinetic theory applied to the shear flows of granular materials

Physics of Fluids 29, 043302 (2017); 10.1063/1.4979632

The lowest Reynolds number of vortex-induced vibrations

Physics of Fluids 29, 041701 (2017); 10.1063/1.4979966

On the shape memory of red blood cells

Physics of Fluids 29, 041901 (2017); 10.1063/1.4979271

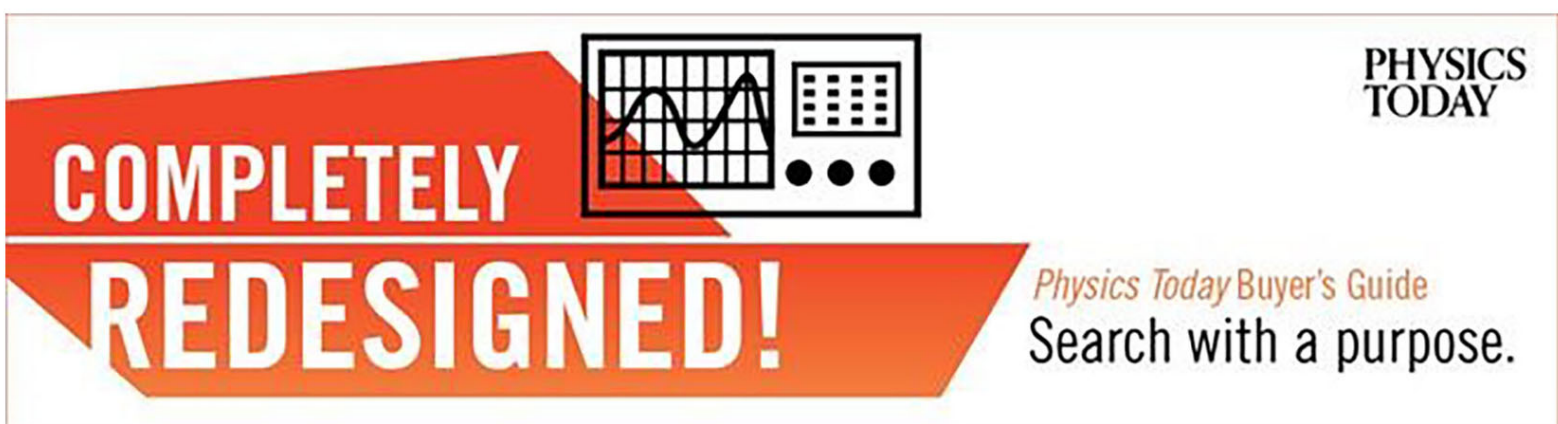




\title{
Asymptotic modeling of thermal binary monatomic gas flows in plane microchannels-Comparison with DSMC simulations
}

\author{
Renée Gatignola ${ }^{\mathrm{a})}$ and Cédric Croizet ${ }^{\mathrm{b})}$ \\ Sorbonne Universités, CNRS, Institut Jean Le Rond d'Alembert, UPMC Univ Paris 06, UMR 7190, \\ F-75005 Paris, France
}

(Received 5 December 2016; accepted 17 March 2017; published online 10 April 2017)

\begin{abstract}
Asymptotic models are constructed to investigate the basic physical phenomena of thermal flows of a mixture of two monatomic gases inside a two-dimensional microchannel. The steady flows are described by the Navier-Stokes-Fourier balance equations, with additional coupling terms in momentum and energy equations, and with first-order slip boundary conditions for the velocities and jump boundary conditions for the temperatures on the two walls. The small parameter equal to the ratio of the two longitudinal and transverse lengths is introduced, and then an asymptotic model is proposed. It corresponds to small Mach numbers and small or moderate Knudsen numbers. Attention is paid to the first-order asymptotic solutions. Results are given and discussed for different cases: the mass flow rates, the molecular weights of the gases, and the temperature gradients along the walls. Comparisons between the first-order asymptotic solutions and Direct Simulation Monte Carlo (DSMC) simulations corresponding to the same physical data show rather good agreement. It should be noted that obtaining an asymptotic solution is very fast compared to obtaining a DSMC result. Published by AIP Publishing. [http://dx.doi.org/10.1063/1.4979683]
\end{abstract}

\section{INTRODUCTION}

Microdevices, whose characteristic dimensions are typically of the order of one micron, are commonly found in modern industry. Examples include micro-mixers, micro-turbines, micro-cooling systems, micro-heat-exchangers, etc. If we consider air flowing in a microtube with a diameter of one micron, at standard pressure and temperature, the mean free path is of the order of $0.1 \mu \mathrm{m} .{ }^{1}$ In this case, the Knudsen number $K n$, equal to the ratio of the mean free path to the diameter of the microtube, is 0.1 . Therefore, the gas is in a rarefied regime. Depending on the Knudsen number, the rarefied flow is said to be in the continuous regime if $0<K n<0.01$, the slipping regime if $0.01<K n<0.1$, the transition regime if $0.1<K n<10$, or the free molecular regime if $K n>10$. ${ }^{2,3}$ Of course, the boundary values of $K n$ which are given to distinguish the different regimes are clearly asymptotic. As an example, in the papers, ${ }^{4-6}$ the separation value between the continuum regime and the slipping regime is $K n$ approximately equal to 0.001 .

There have been numerous works published on the subject of gaseous flows in microchannels or microtubes. The first papers on such flows were published in the field of the Boltzmann equation. ${ }^{1}$ More recently, these flows have been extensively studied over the course of the past twenty years, as they are found in Micro-Electro-Mechanical Systems (MEMS). For a simple isothermal or thermal gas, many theoretical results can be obtained by using the Boltzmann equation ${ }^{1}$ or kinetic equation models, such as the Bhatnagar, Gross and Krook model (BGK model $)^{7}$ or the $\mathrm{S}$ model.$^{8}$ Using

\footnotetext{
a)Electronic mail: renee.gatignol@upmc.fr.

b)Electronic mail: cedric.croizet@upmc.fr
}

the linearized Boltzmann equation, numerical simulations of plane thermal Couette flows have been performed by Ohwada et al. ${ }^{9}$ Sharipov ${ }^{10,11}$ focuses on flows driven by small pressure or temperature gradients, through a straight microchannel with a rectangular cross section. Mass flow is analytically calculated using the BGK equation for isothermal flows ${ }^{10}$ and using the $\mathrm{S}$ model ${ }^{11}$ for thermal flows. Using the $\mathrm{S}$ model kinetic equation, Titarev ${ }^{12}$ constructs an implicit method for calculating rarefied gas flows in a plane microchannel.

Statistical methods such as the Direct Simulation Monte Carlo (DSMC) are useful to study the transition regimes of rarefied gas flows. As is well known in the DSMC methods, the microscopic physics of collisions between molecules and between molecules and walls is statistically described. In the wake of earliest works by Bird, ${ }^{13}$ many papers have been published, with improvements and generalizations of the method, and with many applications, particularly to flows in microchannels and microtubes. ${ }^{2,3,14}$ The lattice Boltzmann methods are also very useful in simulating such flows, especially in the slipping and transition regimes, although as yet, there are few applications to real-world physical problems. ${ }^{15-20}$

DSMC simulations are relevant for describing realistic gas flows in micro-systems, ${ }^{2,3}$ but they are costly in terms of computation time. Hence, for a simple isothermal gas flow in a microchannel or microtube, many authors attempted to justify a continuous approach with balance Navier-Stokes equations and slip boundary conditions on the walls. The goal is to justify the slipping flow regime for the greatest possible Knudsen numbers. ${ }^{2,21}$ In the simplest macroscopic modeling, the flow with slip conditions on the walls is steady, isothermal, and one-dimensional, with the velocity parallel to the longitudinal axis. ${ }^{4,22} \mathrm{~A}$ few analytical results are obtained for the flow through any microchannel, regardless of it cross 
section, rectangular, or trapezoidal. ${ }^{23-25}$ Still, in terms of a flow in microchannels, there are models deduced from the Navier-Stokes equations where the two velocity components, longitudinal and transversal, are both taken into account, ${ }^{26}$ or where the three velocity components are studied. ${ }^{27}$

It is clear that experimental studies of gas flows in microchannels or microtubes, although difficult, are absolutely necessary to validate the results derived from asymptotic or numerical models. The experimental results are mainly related to the pressure distribution along the microchanne $\mathrm{l}^{28}$ and the mass flow rate through the channel..$^{4,21,29}$ Note also that the friction factor relating the friction effects to the Reynolds number in microchannels and microtubes was experimentally investigated. ${ }^{30,31}$

There are fewer papers on the asymptotic modeling of thermal gas flows in two-dimensional microchannels compared to isothermal flows. Temperature and heat-transfer problems are considered by Kuddusi and Çetegen ${ }^{25}$ who use the Navier-Stokes-Fourier equations, by Weng and $\mathrm{Chen}^{32}$ who consider a vertical flow described by the usual Boussinesq approximation, and by Lockerby and Reeve ${ }^{5}$ and Xue et al. $^{33}$ who use the Burnett system. However, in these papers, the flow is assumed to be one-dimensional. In the work of Cai et al. ${ }^{34}$ the gas flow is two-dimensional, and the thermal problem is studied; therefore, the authors assume that the problem is quasi-isothermal so that the temperature is constant as an initial approximation. Using the regularized 13-moment equations, Taheri et al. ${ }^{35}$ proposed an analytical solution for thermal flow in a microchannel.

Now, let us look at flows of a thermal mixture of two gases in the absence of chemical reactions and in the context of rarefied gas dynamics. One possible model of the two-gas mixture corresponds to a single gas characterized by average quantities such as the average density and the average velocity and the boundary conditions on the walls defined for that homogeneous medium. ${ }^{36,37}$ However, we can also consider the mixture as the sum of its two components. Thus, it is described by two coupled non-linear Boltzmann equations. ${ }^{1,38}$ However, it is a very complex task to study them. Therefore, model equations have been put forward in the literature. By performing the linearization of the non-linear Boltzmann equations of the two gases, McCormack ${ }^{39}$ constructed linear kinetic models for the binary gas mixture. This model has been used to theoretically analyze the flows of gas mixture in a microchannel or a microtube in several papers. ${ }^{40-42}$ The flows due to low pressure and concentration gradients, for a binary mixture through long microchannels with trapezoidal and rectangular cross section, are studied in the Szalmas paper. ${ }^{40}$ With the helium-argon mixture, and for pressure-driven flows through long microchannel with rectangular cross section, a comparative study between theoretical and experimental results was carried out. ${ }^{43}$ The authors obtained very good consensus on a large range of Knudsen numbers in the transition regime. This bibliography includes numerical works by Takata et al..$^{44}$ on the two linearized Boltzmann equations, simulations on complex geometries with the conventional DSMC method, ${ }^{2}$ and those performed with modified DSMC method. ${ }^{41}$ We add the simulations obtained with the DS2V code ${ }^{45}$ for flows of a two gas mixture in a microchannel. ${ }^{46}$
From a theoretical point of view, the regularized 17moment equations modeling the binary mixtures of monatomic inert ideal gases in the recent paper of Gupta et $a l^{47}$ should also give interesting results for a flow in a microchannel. In the paper of Rahimi and Struchtrup, ${ }^{48}$ a set of regularized partial differential equations is obtained for a binary rarefied polyatomic gas mixture, where two different relaxation times are introduced to take into account the translational and internal energy exchanges. Associated boundary conditions on a wall are proposed. In the particular case of a mixture at rest, the steady heat conduction normal to two parallel plates is studied.

Following the well-known BGK equation for simple gas, ${ }^{7}$ Sirovich ${ }^{49}$ put forward two generalized BGK equations for the binary mixture of monatomic gases. Both BGK equations are coupled and they possess the main properties of the complete Boltzmann equations regarding the hydrodynamic properties of the medium. Following on from Sirovich's paper, Morse gave precise expressions for eight parameters (six pseudovelocity components and two pseudo-temperatures) included in the model, and he wrote the BGK equations for a binary mixture in a form that is relatively easy to use. ${ }^{50}$ These model equations have been used to study the shock tube problem for argon-neon mixture. ${ }^{51}$ They have also been the basis of quasigasdynamic macroscopic (QGDM) equations for a gas mixture used in the study of a shock wave structure in a helium-xenon mixture. ${ }^{52}$ They are also the basis of the work presented in this paper.

It seems that the flow of a binary gas mixture inside a microchannel has not been studied with these generalized BGK equations. Note also that the heat transfer between the mixture and the microchannel walls must not be neglected in many problems, such as diesel engines and heat exchangers. In this paper, the steady flow of a thermal binary gas mixture in a two-dimensional microchannel is considered. The two walls are at rest and have the same temperature depending only on the longitudinal space variable. The flow of each gas species is described by the usual Navier-Stokes-Fourier balance equations, for mass, momentum, and energy, with additional coupling terms in momentum and energy equations deduced from kinetic BGK models. On the walls, the firstorder slip conditions for the velocities and the jump conditions for the temperatures are written.

In Section II, for each gas, the Navier-Stokes-Fourier balance equations are deduced from the kinetic BGK models for the mixture. In Section III, with the transverse and longitudinal characteristic length scales of the microchannel, a small parameter $\varepsilon$ is introduced. Using the assumptions that, for each gas, the Mach number and the Knudsen number are small or of order unity, we construct an asymptotic model with the Mach numbers of order $O(\varepsilon)$ and the Knudsen numbers of order $O\left(\varepsilon^{0}\right)$. This is due to the principle of least degeneracy. ${ }^{53}$ The asymptotic approximation at order zero in $\varepsilon$ is presented in Section IV. Then, in Section V, the numerical results at order zero in $\varepsilon$ are given and discussed, in light of different parameters: the two mass flow rates, the molecular weights of the gases, and the temperature gradient along the walls. Additionally, in Section VI, we compare the first approximation solutions with DSMC simulations. We verify that there is rather good agreement between the asymptotic and DSMC 
results for the studied cases where the average Knudsen number is between 0.16 and 0.19 , and the average Mach number is of order of 0.016 .

\section{PROBLEM EQUATIONS}

Consider a plane microchannel having the length $L$ and the width $h$. An orthonormal frame $\left(O ; x^{*}, y^{*}\right)$ is introduced with the point $O$ at the center of the inlet section $\left(x^{*}=0\right)$ of the microchannel and the $x^{*}$-axis parallel to the two walls. A mixture of two gas species denoted by $a$ and $b$, without chemical reactions, is contained inside the channel. Without volumetric force and heat source, the gases flow due to the influence of pressure and concentration gradients and temperature gradients along the walls. As a consequence of the small dimensions of the microchannel, the two gas flows are rarefied. Our purpose is to introduce models to describe the flows of these two gases. Throughout this paper, the asterisk exponent is placed on all dimension quantities (see short nomenclature), except for quantities which are constant, such as the molecular weights $m_{a}$ and $m_{b}$ of the two species. First, we assume that the two species are described by two kinetic BGK-type equations ${ }^{50-52}$

$$
\begin{aligned}
& \frac{\partial f_{a}^{*}}{\partial t^{*}}+\xi^{*} \cdot \frac{\partial f_{a}^{*}}{\partial \mathbf{x}^{*}}=v_{a}^{*}\left(F_{a}^{*}-f_{a}^{*}\right)+v_{a b}^{*}\left(\bar{F}_{a}^{*}-f_{a}^{*}\right), \\
& \frac{\partial f_{b}^{*}}{\partial t^{*}}+\xi^{*} \cdot \frac{\partial f_{b}^{*}}{\partial \mathbf{x}^{*}}=v_{b}^{*}\left(F_{b}^{*}-f_{b}^{*}\right)+v_{b a}^{*}\left(\bar{F}_{b}^{*}-f_{b}^{*}\right),
\end{aligned}
$$

where $f_{a}^{*}\left(t^{*}, \mathbf{x}^{*}, \xi^{*}\right)$ and $f_{b}^{*}\left(t^{*}, \mathbf{x}^{*}, \xi^{*}\right)$ are the velocity distribution functions for species $a$ and $b$, at time $t^{*}$ and point $\mathbf{x}^{*}$ and where the molecule velocity is denoted by $\xi^{*}$. In Eqs. (1) and (2), $v_{a}^{*}$ and $v_{b}^{*}$ are the frequencies of self-collisions between the molecules $a$ and the molecules $b, v_{a b}^{*}$ the frequency of cross-collisions of the molecules $b$ with a molecule $a$, and $v_{b a}^{*}$ the frequency of cross-collisions of the molecules $a$ with a molecule $b$. The total number of collisions between the two species should be balanced, so we have $n_{a}^{*} v_{a b}^{*}=n_{b}^{*} v_{b a}^{*}$, where $n_{a}^{*}$ and $n_{b}^{*}$ are the number densities of the species $a$ and $b$.

To simplify the presentation and in the absence of any ambiguity, we introduce the notation $\theta$ to designate one or the other of the two gases: $\theta=a$ or $b$. In Eqs. (1) and (2), $F_{\theta}^{*}$ is the Maxwellian distribution function defined with the number density $n_{\theta}^{*}$, the mean velocity $\mathbf{u}_{\theta}^{*}$, and the temperature $T_{\theta}^{*}$ of the species $\theta$, and $\bar{F}_{\theta}^{*}$ is the Maxwellian distribution function defined with $n_{\theta}^{*}$ and with the pseudo-velocity $\overline{\mathbf{u}}_{\theta}^{*}$ and the pseudo-temperature $\bar{T}_{\theta}^{*}$ of the species $\theta$ defined as follows: $:^{50-52}$

$$
\begin{gathered}
F_{\theta}^{*}=\frac{n_{\theta}^{*}}{\left(2 \pi r_{\theta} T_{\theta}^{*}\right)^{3 / 2}} \exp \left(\frac{-\left(\xi^{*}-\mathbf{u}_{\theta}^{*}\right)^{2}}{2 r_{\theta} T_{\theta}^{*}}\right), \\
\bar{F}_{\theta}^{*}=\frac{n_{\theta}^{*}}{\left(2 \pi r_{\theta} \bar{T}_{\theta}^{*}\right)^{3 / 2}} \exp \left(\frac{-\left(\xi^{*}-\overline{\mathbf{u}}_{\theta}^{*}\right)^{2}}{2 r_{\theta} \bar{T}_{\theta}^{*}}\right), \\
\overline{\mathbf{u}}_{a}^{*}=\overline{\mathbf{u}}_{b}^{*}=\frac{m_{a} \mathbf{u}_{a}^{*}+m_{b} \mathbf{u}_{b}^{*}}{m_{a}+m_{b}},
\end{gathered}
$$

$$
\begin{aligned}
& \bar{T}_{a}^{*}=T_{a}^{*}+\frac{2 m_{a} m_{b}}{\left(m_{a}+m_{b}\right)^{2}}\left(T_{b}^{*}-T_{a}^{*}+\frac{m_{b}}{6 k}\left(\mathbf{u}_{b}^{*}-\mathbf{u}_{a}^{*}\right)^{2}\right), \\
& \bar{T}_{b}^{*}=T_{b}^{*}+\frac{2 m_{a} m_{b}}{\left(m_{a}+m_{b}\right)^{2}}\left(T_{a}^{*}-T_{b}^{*}+\frac{m_{a}}{6 k}\left(\mathbf{u}_{a}^{*}-\mathbf{u}_{b}^{*}\right)^{2}\right),
\end{aligned}
$$

where $k$ is the Boltzmann constant and $r_{\theta}=k / m_{\theta}$. Note that $\overline{\mathbf{u}}_{a}^{*}$ and $\overline{\mathbf{u}}_{b}^{*}$ are the barycentric velocity of the mixture. ${ }^{50,52}$ Additionally, each gas species is an ideal gas with constant massic heat capacities $c_{p \theta}$ and $c_{v \theta}$; then $p_{\theta}^{*}=r_{\theta} \rho_{\theta}^{*} T_{\theta}^{*}, c_{v \theta}$ $=r_{\theta} /\left(\gamma_{\theta}-1\right), c_{p \theta}=\gamma_{\theta} r_{\theta} /\left(\gamma_{\theta}-1\right)$, where $\gamma_{\theta}=c_{p \theta} / c_{v \theta}$. In this paper, only cases where the gases $a$ and $b$ are monatomic are considered. That is to say $\gamma_{\theta}=5 / 3, c_{v \theta}=(3 / 2) r_{\theta}$, and $c_{p \theta}=(5 / 2) r_{\theta}$. This assumption is taken into account in expressions (5) and (6) for $\bar{T}_{a}^{*}$ and $\bar{T}_{b}^{*}$. Expressions (5) and (6) are equal to the expressions given by Morse ${ }^{50}$ and by Elizarova et al. ${ }^{52}$

The definitions of the macroscopic quantities of the gas species $\theta$ according to the distribution function $f_{\theta}^{*}$ are well known. ${ }^{1,38}$ To clarify the notations, let us recap a number of formulae

$$
\begin{gathered}
m_{\theta} \int f_{\theta}^{*} d \xi^{*}=m_{\theta} n_{\theta}^{*}=\rho_{\theta}^{*}, \quad m_{\theta} \int \xi^{*} f_{\theta}^{*} d \xi^{*}=\rho_{\theta}^{*} \mathbf{u}_{\theta}^{*}, \\
m_{\theta} \int \mathbf{c}_{\theta}^{*} \mathbf{c}_{\theta}^{*} f_{\theta}^{*} d \xi^{*}=-\tau_{\theta}^{*}+p_{\theta}^{*} I_{i d}, \\
\frac{1}{2} m_{\theta} \int \mathbf{c}_{\theta}^{*}\left(\mathbf{c}_{\theta}^{*}\right)^{2} f_{\theta}^{*} d \xi^{*}=\mathbf{q}_{\theta}^{*}, \\
\frac{1}{2} m_{\theta} \int\left(\mathbf{c}_{\theta}^{*}\right)^{2} f_{\theta}^{*} d \xi^{*}=\frac{3}{2} r_{\theta} \rho_{\theta}^{*} T_{\theta}^{*}=\frac{3}{2} p_{\theta}^{*}
\end{gathered}
$$

with $\mathbf{c}_{\theta}^{*}=\xi-\mathbf{u}_{\theta}^{*}$ and where $\rho_{\theta}^{*}$ is the mass of the unit volume, $p_{\theta}^{*}$ the pressure, $\tau_{\theta}^{*}$ the viscous stress tensor, $I_{i d}$ the tensor unity, and $\mathbf{q}_{\theta}^{*}$ the heat flux vector. To obtain the macroscopic balance equations for mass, momentum, and total energy, each of Equation (1) or (2) is multiplied by $m_{\theta}, m_{\theta} \xi^{*}$, and $1 / 2 m_{\theta} \xi^{* 2}$. After integration over the space of molecular velocities, the set of balance equations for steady flows is ${ }^{52}$

$$
\begin{gathered}
\frac{\partial}{\partial \mathbf{x}^{*}} \cdot\left(\rho_{\theta}^{*} \mathbf{u}_{\theta}^{*}\right)=0 \\
\frac{\partial}{\partial \mathbf{x}^{*}} \cdot\left(\rho_{\theta}^{*} \mathbf{u}_{\theta}^{*} \mathbf{u}_{\theta}^{*}\right)+\frac{\partial p_{\theta}^{*}}{\partial \mathbf{x}^{*}}-\frac{\partial}{\partial \mathbf{x}^{*}} \cdot \tau_{\theta}^{*}=\mathbf{S}_{\theta}^{\mathbf{u}^{*}} \\
\frac{\partial}{\partial \mathbf{x}^{*}} \cdot\left(\rho_{\theta}^{*}\left(e_{\theta}^{*}+\frac{1}{2}\left(\mathbf{u}_{\theta}^{*}\right)^{2}\right) \mathbf{u}_{\theta}^{*}\right) \\
+\frac{\partial}{\partial \mathbf{x}^{*}} \cdot\left(p_{\theta}^{*} \mathbf{u}_{\theta}^{*}-\tau_{\theta}^{*} \cdot \mathbf{u}_{\theta}^{*}+\mathbf{q}_{\theta}^{*}\right)=S_{\theta}^{E^{*}}
\end{gathered}
$$

In Eq. (10), the internal energy per unit mass, $e_{\theta}^{*}$, is introduced: $e_{\theta}^{*}=c_{v \theta} T_{\theta}^{*}=(3 / 2) r_{\theta} T_{\theta}^{*}$ for monatomic gases, and $e_{\theta}^{*}=r_{\theta} T_{\theta}^{*} /\left(\gamma_{\theta}-1\right)$ in the general case of polyatomic gases. The coupling terms in the balance laws for momentum and energy, $\mathbf{S}_{\theta}^{\mathbf{u}^{*}}$ and $S_{\theta}^{E^{*}}$ are easy to explicitly compute

$$
\begin{gathered}
\mathbf{S}_{a}^{\mathbf{u}^{*}}=v_{a b}^{*} \rho_{a}^{*}\left(\overline{\mathbf{u}}_{a}^{*}-\mathbf{u}_{a}^{*}\right) \\
S_{a}^{E^{*}}=v_{a b}^{*} \rho_{a}^{*}\left[\frac{3}{2} r_{a}\left(\bar{T}_{a}^{*}-T_{a}^{*}\right)+\frac{1}{2}\left(\overline{\mathbf{u}}_{a}^{* 2}-\mathbf{u}_{a}^{* 2}\right)\right] .
\end{gathered}
$$


Analogous expressions for $\mathbf{S}_{b}^{\mathbf{u}^{*}}$ and $S_{b}^{E^{*}}$ are obtained by exchanging the two species $a$ and $b$. Of course: $\mathbf{S}_{a}^{\mathbf{u}^{*}}+\mathbf{S}_{b}^{\mathbf{u}^{*}}=0$ and $S_{a}^{E^{*}}+S_{b}^{E^{*}}=0$. It should be noted that these relations are consequences of the definitions (4)-(6). In the case of a mixture of non-monatomic gases, the definitions (5) and (6) need to be modified (see Appendix A).

Equations (9) and (10) are now written in a simpler form. The temperature $T_{\theta}^{*}$ (see Eq. (8)) and the constitutive laws are introduced

$$
\begin{gathered}
\frac{\partial}{\partial \mathbf{x}^{*}} \cdot\left(\rho_{\theta}^{*} \mathbf{u}_{\theta}^{*}\right)=0, \quad \rho_{\theta}^{*} \mathbf{u}_{\theta}^{*} \cdot \frac{\partial \mathbf{u}_{\theta}^{*}}{\partial \mathbf{x}^{*}}+\frac{\partial p_{\theta}^{*}}{\partial \mathbf{x}^{*}}-\frac{\partial}{\partial \mathbf{x}^{*}} \cdot \tau_{\theta}^{*}=\mathbf{S}_{\theta}^{\mathbf{u}^{*}}, \\
\rho_{\theta}^{*} \frac{1}{\gamma_{\theta}-1} r_{\theta} \mathbf{u}_{\theta}^{*} \cdot \frac{\partial T_{\theta}^{*}}{\partial \mathbf{x}^{*}}+p_{\theta}^{*} \frac{\partial}{\partial \mathbf{x}^{*}} \cdot \mathbf{u}_{\theta}^{*}-\tau_{\theta}^{*}: \frac{\partial \mathbf{u}_{\theta}^{*}}{\partial \mathbf{x}^{*}} \\
+\frac{\partial \mathbf{q}_{\theta}^{*}}{\partial \mathbf{x}^{*}}=S_{\theta}^{E^{*}}-\mathbf{S}_{\theta}^{\mathbf{u}^{*}} \cdot \mathbf{u}_{\theta}^{*}, \\
\tau_{\theta i j}^{*}=\mu_{\theta}^{*}\left(\frac{\partial u_{\theta i}^{*}}{\partial x_{j}^{*}}+\frac{\partial u_{\theta j}^{*}}{\partial x_{i}^{*}}\right)+\lambda_{\theta}^{*} \frac{\partial u_{\theta \ell}^{*}}{\partial x_{\ell}^{*}} \delta_{i j}, \quad q_{\theta j}^{*}=-\kappa_{\theta}^{*} \frac{\partial T_{\theta}^{*}}{\partial x_{j}^{*}}
\end{gathered}
$$

In Eq. (14), the Einstein convention is used with $i, j, \ell$ equal to 1,2 or 3 . The transport coefficients $\mu_{\theta}^{*}$ (shear viscosity), $\lambda_{\theta}^{*}$ (expansion viscosity), and $\kappa_{\theta}^{*}$ (thermal conductivity) are positive and are assumed to depend solely on the temperature $T_{\theta}^{*}$. Additionally, the two coefficients $\mu_{\theta}^{*}$ and $\kappa_{\theta}^{*}$ are such that the Prandtl number $\operatorname{Pr}_{\theta}=c_{p \theta} \mu_{\theta}^{*} / \kappa_{\theta}^{*}$ of the species $\theta$ is constant. From kinetic theory for monatomic gases, we know that $\operatorname{Pr}_{\theta}$ is equal to 2/3. ${ }^{1,54}$ The right member of Eq. (13) for the gas species $a$ is given in Eq. (15). A similar relation can be written for component $b$ by exchanging $a$ and $b$

$$
\begin{aligned}
S_{a}^{E^{*}}-\mathbf{S}_{a}^{\mathbf{u}^{*}} \cdot \mathbf{u}_{a}^{*}= & v_{a b}^{*} n_{a}^{*} \frac{2 m_{a} m_{b}}{\left(m_{a}+m_{b}\right)^{2}}\left(\frac{3 k}{2}\left(T_{b}^{*}-T_{a}^{*}\right)\right. \\
& \left.+\frac{m_{b}}{2}\left(\mathbf{u}_{b}^{*}-\mathbf{u}_{a}^{*}\right)^{2}\right) .
\end{aligned}
$$

Let us consider the two gases flowing in the microchannel. In the frame $\left(O, x^{*}, y^{*}\right)$, the two components of the velocity $\mathbf{u}_{\theta}^{*}$ are denoted by $u_{\theta}^{*}$ and $v_{\theta}^{*}$. The walls located at $y^{*}= \pm h / 2$ are at rest and have the same temperature $T_{w}^{*}\left(x^{*}\right)$. The first-order slip boundary conditions for the velocities and the jump conditions for the temperatures at $y^{*}= \pm h / 2$ are given as follows: ${ }^{37}$

$$
\begin{gathered}
u_{\theta}^{*}=\mp \sigma_{\theta P} \sqrt{2 r_{\theta} T_{\theta}^{*}} \frac{\mu_{\theta}^{*}}{p_{\theta}^{*}} \frac{\partial u_{\theta}^{*}}{\partial y^{*}}+\sigma_{\theta T} r_{\theta} \frac{\mu_{\theta}^{*}}{p_{\theta}^{*}} \frac{\partial T_{\theta}^{*}}{\partial x^{*}}, \quad v_{\theta}^{*}=0, \\
T_{\theta}^{*}=T_{w}^{*} \mp \zeta_{\theta T} \sqrt{2 r_{\theta} T_{\theta}^{*}} \frac{\mu_{\theta}^{*}}{p_{\theta}^{*}} \frac{\partial T_{\theta}^{*}}{\partial y^{*}} .
\end{gathered}
$$

The coefficients $\sigma_{\theta P}$ and $\sigma_{\theta T}$ characterize the viscous and thermal slip of the species $\theta$ on the walls, and $\zeta_{\theta T}$ the jump temperature of the species $\theta$ on the walls. ${ }^{37}$ In the present paper, these three coefficients are constant. The upstream and downstream boundary conditions for the pressure will be specified later on. Finally, we give explicit expressions for the collision frequencies. Following Bird, ${ }^{54}$ we set

$$
\begin{gathered}
v_{\theta}^{*}=\frac{p_{\theta}^{*}}{\mu_{\theta}^{*}} \Omega\left(\omega_{\theta}, \alpha_{\theta}\right), \quad \mu_{\theta}^{*}=\mu_{\theta}^{r e f}\left(\frac{T_{\theta}^{*}}{T_{\theta}^{r e f}}\right)^{\omega_{\theta}}, \\
\Omega_{\theta} \equiv \Omega\left(\omega_{\theta}, \alpha_{\theta}\right)=\frac{5\left(\alpha_{\theta}+1\right)\left(\alpha_{\theta}+2\right)}{\alpha_{\theta}\left(7-2 \omega_{\theta}\right)\left(5-2 \omega_{\theta}\right)},
\end{gathered}
$$

where $\omega_{\theta}$ is the viscosity index and $\alpha_{\theta}$ the variable soft sphere (VSS) parameter. ${ }^{46,54,55}$ For the cross frequencies $v_{a b}^{*}$ and $v_{b a}^{*}$ between the molecules $a$ and $b$, we write expressions similar to those of $\mathrm{Wu}$ and $\mathrm{Lee}^{51}$ and Reyhanian ${ }^{55}$

$$
\begin{aligned}
& v_{a b}^{*}=v_{a}^{*} \frac{n_{b}^{*}}{n_{a}^{*}}\left(\frac{d_{a}+d_{b}}{2 d_{a}}\right)^{2}\left(\frac{m_{a}+m_{b}}{2 m_{b}}\right)^{1 / 2} \psi_{a b}^{*}, \\
& v_{b a}^{*}=v_{b}^{*} \frac{n_{a}^{*}}{n_{b}^{*}}\left(\frac{d_{a}+d_{b}}{2 d_{b}}\right)^{2}\left(\frac{m_{a}+m_{b}}{2 m_{a}}\right)^{1 / 2} \psi_{b a}^{*},
\end{aligned}
$$

where $\psi_{a b}^{*}$ and $\psi_{b a}^{*}$ must be modeled. As previously stated, the collision frequency between the molecules $a$ and $b$ is equal to the collision frequency between the molecules $b$ and $a$, i.e., $v_{a b}^{*} n_{a}^{*}=v_{b a}^{*} n_{b}^{*}$. Consequently, the following relation must be verified:

$$
\frac{\Omega_{a} T_{a}^{*}}{\mu_{a}^{*}} \frac{m_{a}^{1 / 2}}{d_{a}^{2}} \psi_{a b}^{*}=\frac{\Omega_{b} T_{b}^{*}}{\mu_{b}^{*}} \frac{m_{b}^{1 / 2}}{d_{b}^{2}} \psi_{b a}^{*} .
$$

In her thesis, ${ }^{55}$ Reyhanian introduced the following model for $v_{a b}^{*}$ and $v_{b a}^{*}$ with $\psi_{a b}^{*}=1$ and $\psi_{b a}^{*}$ given by relation (19). The two species do not appear symmetrically (the expression of $v_{b a}^{*}$ cannot be deduced from that of $v_{a b}^{*}$ by exchanging $a$ and $b$ ). Another possible option would be to set $\psi_{a b}^{*}$ $=\psi_{b a}^{*}=1$, but then relation (19) is not satisfied. Here, we set $\beta_{\theta}^{*}=\left(m_{\theta}^{1 / 2} \Omega_{\theta} T_{\theta}^{*}\right) /\left(d_{\theta}^{2} \mu_{\theta}^{*}\right)$, and we propose (this choice will be discussed in Section V A)

$$
\psi_{a b}^{*}=\left(\frac{\beta_{b}^{*}}{\beta_{a}^{*}}\right)^{1 / 2}, \quad \psi_{b a}^{*}=\left(\frac{\beta_{a}^{*}}{\beta_{b}^{*}}\right)^{1 / 2} .
$$

\section{ASYMPTOTIC MODEL EQUATIONS}

From now on, the steady flows of both gases inside the plane microchannel are considered. All the unknown functions $u_{\theta}^{*}, v_{\theta}^{*}, \rho_{\theta}^{*}, p_{\theta}^{*}$, and $T_{\theta}^{*}$ are assumed to be dependent only on the spatial variables $x^{*}$ and $y^{*}$. The goal is to discuss the order of magnitude of the different terms present in the equations. First, Eqs. (12) and (13) are written in dimensionless form, and to do so, characteristic values are introduced. The chosen characteristic scales for the longitudinal and transverse lengths, the longitudinal and transverse velocities, the pressures, the temperatures, the volumetric masses, the shear viscosities, the expansion viscosities, and the thermal conductivities are, respectively, $L, h, U_{c}, V_{c}, P_{c}$, and $T_{c}$ (for the two species and the walls), $\rho_{\theta c}$ (with the ideal gas law $P_{c}=r_{\theta} \rho_{\theta c} T_{c}$ ), $\mu_{\theta c}, \lambda_{\theta c}$, and $\kappa_{\theta c}$ (with the Prandtl number $\operatorname{Pr}_{\theta}$ equal to $\left.\left(c_{p \theta} \mu_{\theta c}\right) / \kappa_{\theta c}\right)$. The dimensionless quantities are $x=x^{*} / L, y=y^{*} / h, u_{\theta}=u_{\theta}^{*} / U_{c}, v_{\theta}=v_{\theta}^{*} / V_{c}, p_{\theta}=p_{\theta}^{*} / P_{c}$, $T_{\theta}=T_{\theta}^{*} / T_{c}, \rho_{\theta}=\rho_{\theta}{ }^{*} / \rho_{\theta c}, \mu_{\theta}=\mu_{\theta}^{*} / \mu_{\theta c}, \lambda_{\theta}=\lambda_{\theta}^{*} / \lambda_{\theta c}$, and $\kappa_{\theta}=\kappa_{\theta}^{*} / \kappa_{\theta c}$. Here, we list all dimensionless numbers that will appear in the dimensionless balance equations, namely, the small parameter $\varepsilon$, the heat capacity ratio $\gamma_{\theta}$, the Mach number $M_{\theta}$, the Knudsen number $K n_{\theta}$, and the Reynolds number $\operatorname{Re}_{\theta}$ 


$$
\begin{gathered}
\varepsilon=\frac{h}{L}, \quad \gamma_{\theta}=\frac{c_{p \theta}}{c_{v \theta}}=\frac{5}{3}, \quad M_{\theta}=\frac{U_{c}}{\sqrt{\gamma_{\theta} r_{\theta} T_{c}}}, \\
K n_{\theta}=\frac{1}{h} \frac{\mu_{\theta c}}{\rho_{\theta c}} \sqrt{\frac{\pi}{2 r_{\theta} T_{c}}}=\frac{1}{h} \frac{\mu_{\theta c}}{P_{c}} \sqrt{\frac{\pi r_{\theta} T_{c}}{2}}, \\
R e_{\theta}=\frac{\rho_{\theta c} U_{c} h}{\mu_{\theta c}}=\frac{M_{\theta}}{K n_{\theta}} \sqrt{\frac{\pi \gamma_{\theta}}{2}} .
\end{gathered}
$$

Due to the geometry of the microchannel, we assume $\varepsilon \ll 1$. As a result of the principle of least degeneracy, ${ }^{53}$ we must keep the two terms in balance equations for mass. So $V_{c}=\varepsilon U_{c}$. Now, the dimensionless equations deduced from Eqs. (12) and (13) are written as follows:

$$
\frac{\partial \rho_{\theta} u_{\theta}}{\partial x}+\frac{\partial \rho_{\theta} v_{\theta}}{\partial y}=0
$$

$$
\begin{aligned}
& \rho_{\theta}\left(u_{\theta} \frac{\partial T_{\theta}}{\partial x}+v_{\theta} \frac{\partial T_{\theta}}{\partial y}\right)-\varepsilon \sqrt{\frac{2 \gamma_{\theta}}{\pi}} \frac{K n_{\theta}}{M_{\theta}} \frac{1}{\operatorname{Pr}_{\theta}}\left(\frac{\partial}{\partial x}\left(\kappa_{\theta} \frac{\partial T_{\theta}}{\partial x}\right)+\frac{1}{\varepsilon^{2}} \frac{\partial}{\partial y}\left(\kappa_{\theta} \frac{\partial T_{\theta}}{\partial y}\right)\right) \\
& +\left(\gamma_{\theta}-1\right) p_{\theta}\left(\frac{\partial u_{\theta}}{\partial x}+\frac{\partial v_{\theta}}{\partial y}\right)-\varepsilon\left(\gamma_{\theta}-1\right) \sqrt{\frac{2 \gamma_{\theta}}{\pi}} M_{\theta} K n_{\theta}\left[\left(\frac{\lambda_{\theta c}}{\mu_{\theta c}} \lambda_{\theta}+2 \mu_{\theta}\right)\left(\left(\frac{\partial u_{\theta}}{\partial x}\right)^{2}+\left(\frac{\partial v_{\theta}}{\partial y}\right)^{2}\right)\right. \\
& \left.+2 \frac{\lambda_{\theta c}}{\mu_{\theta c}} \lambda_{\theta} \frac{\partial u_{\theta}}{\partial x} \frac{\partial v_{\theta}}{\partial y}+\mu_{\theta}\left(\frac{1}{\varepsilon} \frac{\partial u_{\theta}}{\partial y}+\varepsilon \frac{\partial v_{\theta}}{\partial x}\right)^{2}\right]=\tilde{S}_{\theta}^{E} .
\end{aligned}
$$

Hereafter, the right-hand sides of the two Equations (23) and (24) are given in the case of the species $a$ (for the species $b$, the two indices $a$ and $b$ are exchanged)

$$
\begin{aligned}
\tilde{S}_{a}^{u} & =\frac{1}{\varepsilon} F_{a b} \frac{M_{a}}{K n_{a}} \Omega_{a} \psi_{a b} \frac{p_{a} \rho_{b}}{\mu_{a}}\left(u_{b}-u_{a}\right), \\
\tilde{S}_{a}^{v} & =\frac{1}{\varepsilon} F_{a b} \frac{M_{a}}{K n_{a}} \Omega_{a} \psi_{a b} \frac{p_{a} \rho_{b}}{\mu_{a}}\left(v_{b}-v_{a}\right)
\end{aligned}
$$

with

$F_{a b}=\sqrt{\frac{\pi \gamma_{a}}{2}} \frac{\rho_{b c}}{\rho_{a c}}\left(\frac{d_{a}+d_{b}}{2 d_{a}}\right)^{2} \frac{m_{a}}{\sqrt{2 m_{b}\left(m_{a}+m_{b}\right)}}\left(\psi_{a b}\right)_{c}$,

where $\psi_{a b}^{*}=\left(\psi_{a b}\right)_{c} \psi_{a b}$ and $\left(\psi_{a b}\right)_{c}$ being constant scale that will be specified later, at the end of this section. Remember that expression (15) is correct only for monatomic gases with $\gamma_{a}=\gamma_{b}=5 / 3$. The right-hand side $\tilde{S}_{a}^{E}$ of Eq. (25) is given below

$$
\begin{aligned}
\tilde{S}_{a}^{E}= & \frac{2}{3 \varepsilon} F_{a b} \frac{M_{a}}{K n_{a}} \frac{m_{b}}{m_{a}+m_{b}} \Omega_{a} \psi_{a b} \frac{p_{a} \rho_{b}}{\mu_{a}} \\
& \times\left(\frac{9}{5 M_{b}^{2}}\left(T_{b}-T_{a}\right)+\left(u_{b}-u_{a}\right)^{2}+\varepsilon^{2}\left(v_{b}-v_{a}\right)^{2}\right) .
\end{aligned}
$$

In order to find asymptotic systems, we assume that $M_{\theta}$ and $K n_{\theta}$ are small or of order unity. More precisely, we assume $M_{\theta}=O\left(\varepsilon^{\alpha}\right)$ and $K n_{\theta}=O\left(\varepsilon^{\beta}\right)$ with $\alpha \geq 0$ and $\beta \geq 0$. Of course, $\operatorname{Re}_{\theta}=O\left(\varepsilon^{\alpha-\beta}\right)$; we assume $\alpha-\beta \geq 0$ corresponding to low or moderate Reynolds numbers which is a reasonable assumption in microsystem flows. From the principle of least degeneracy ${ }^{53}$ and taking into account the physics of the problem, we set $\alpha=1$ and $\beta=0$ (Appendix B). This case is interesting from the point of view of the physics of the problem. Indeed, in Eq. (23), the term associated with the pressure gradient is kept, as is the coupling term on the right. The pressure gradient corresponds to a driving force for the flow in the microchannel. Note that the terms present in the energy equation are those of order $\varepsilon^{-2}$. Accordingly, the coupling term is retained. This solution is underlined in the Refs. 55-57 for an isothermal binary gas mixture. It is also introduced in the case of an isothermal single gas in Ref. 26, and in Cai et al., ${ }^{34}$ where it is denoted by case 2A. In the last paper, it is assumed that the inlet pressure is greater than the outlet pressure in order to drive the flow and that the flow temperature experiences small variations. Here, these assumptions are not used: the order of magnitude for the inlet and outlet pressures is $P_{c}$ and for the temperature it is $T_{c}$. In our problem, $P_{c}$ and $T_{c}$ are chosen so that the dimensionless quantities $p_{\theta}$ and $T_{\theta}$ are of order unity.

This case, $\alpha=1$ and $\beta=0$, corresponds to small Mach number and Knudsen number of order unity. We set $M_{\theta}=$ $\varepsilon \sqrt{\pi /\left(2 \gamma_{\theta}\right)} M_{\theta c}$ and $K n_{\theta}=K n_{\theta c}$ with the both numbers $M_{\theta c}$ and $K n_{\theta c}$ of order unity. As an initial approximation, we have the following dimensionless equations for the mass, the 
longitudinal and transversal momentum, and the energy:

$$
\begin{aligned}
& \frac{\partial\left(\rho_{\theta} u_{\theta}\right)}{\partial x}+\frac{\partial\left(\rho_{\theta} v_{\theta}\right)}{\partial y}=0, \\
& \frac{\partial p_{\theta}}{\partial x}-M_{\theta c} K n_{\theta c} \frac{\partial}{\partial y}\left(\mu_{\theta} \frac{\partial u_{\theta}}{\partial y}\right)=\hat{S}_{\theta}^{u}, \quad \frac{\partial p_{\theta}}{\partial y}=0, \\
& -\frac{2 \gamma_{\theta}}{\pi} \frac{K n_{\theta c}}{M_{\theta c}} \frac{1}{\operatorname{Pr}_{\theta}} \frac{\partial}{\partial y}\left(\kappa_{\theta} \frac{\partial T_{\theta}}{\partial y}\right)=\hat{S_{\theta}^{E}}, \\
& \hat{S_{a}^{u}}=F_{a b} \sqrt{\frac{\pi}{2 \gamma_{a}}} \frac{M_{a c}}{K n_{a c}} \Omega_{a} \psi_{a b} \frac{p_{a} \rho_{b}}{\mu_{a}}\left(u_{b}-u_{a}\right), \\
& \hat{S_{a}^{E}}=2 F_{a b} \sqrt{\frac{2}{\pi \gamma_{a}}} \frac{M_{a c}}{M_{b c}^{2} K n_{a c}} \frac{m_{b}}{m_{a}+m_{b}} \Omega_{a} \psi_{a b} \frac{p_{a} \rho_{b}}{\mu_{a}}\left(T_{b}-T_{a}\right)
\end{aligned}
$$

with $\gamma_{\theta}=5 / 3$ and with the ideal gas law $p_{\theta}=\rho_{\theta} T_{\theta}$. We have similar expressions for $\hat{S}_{b}^{u}$ and $\hat{S_{b}^{E}}$ by exchanging the two species $a$ and $b$.

These partial differential equations must be supplemented by boundary conditions. From Eq. (16), with $T_{w}^{*}\left(x^{*}\right)$ $=T_{c} T_{w}(x)$, and the numbers $M_{\theta c}$ and $K n_{\theta c}$ just defined, the dimensionless boundary conditions, in $y= \pm 1 / 2$, are

$$
\begin{gathered}
u_{\theta}=\mp \sigma_{\theta P} \frac{2 K n_{\theta c}}{\sqrt{\pi}} \sqrt{T_{\theta}} \frac{\mu_{\theta}}{p_{\theta}} \frac{\partial u_{\theta}}{\partial y}+\sigma_{\theta T} \frac{2}{\pi} \frac{K n_{\theta c}}{M_{\theta c}} \frac{\mu_{\theta}}{p_{\theta}} \frac{\partial T_{\theta}}{\partial x}, \\
v_{\theta}=0, \quad T_{\theta}=T_{w} \mp \zeta_{\theta T} \frac{2 K n_{\theta c}}{\sqrt{\pi}} \sqrt{T_{\theta}} \frac{\mu_{\theta}}{p_{\theta}} \frac{\partial T_{\theta}}{\partial y} .
\end{gathered}
$$

Note that $K n_{\theta c} \sqrt{T_{\theta}} \mu_{\theta} / p_{\theta}$ corresponds to the local Knudsen number of the gas $\theta$.

Now, we need to give analytical expressions for the two quantities $\psi_{a b}$ and $\psi_{b a}$. The modeling (20) is introduced. With the previous dimensionless variables, we have

$$
\begin{aligned}
\psi_{a b}^{*} & =\left(\frac{\beta_{b}^{*}}{\beta_{a}^{*}}\right)^{1 / 2}=\left(\sqrt{\frac{m_{b}}{m_{a}}} \frac{d_{a}^{2}}{d_{b}^{2}} \frac{\mu_{a c}}{\mu_{b c}}\right)^{1 / 2}\left(\frac{\Omega_{b}}{\Omega_{a}} \frac{T_{b}}{T_{a}} \frac{\mu_{a}}{\mu_{b}}\right)^{1 / 2} \\
& =\left(\psi_{a b}\right)_{c} \psi_{a b},
\end{aligned}
$$

where $\left(\psi_{a b}\right)_{c}$ and $\psi_{a b}$ are, respectively, the first and second factor in Eq. (35). Of course, we can write similar expressions for $\left(\psi_{b a}\right)_{c}$ and $\psi_{b a}$ by exchanging the indices $a$ and $b$. Finally, it is easy to insert this expression for $\left(\psi_{a b}\right)_{c}$ in the definition (27) of $F_{a b}$ and $\psi_{a b}$ in the coupling terms $\hat{S}_{a}^{E}$ and $\hat{S}_{a}^{u}$ (see (32) and (33)).

\section{FIRST-ORDER SOLUTION}

From Eq. (30), the pressures $p_{a}$ and $p_{b}$ depend on $x$ only. First, we are looking for the temperatures $T_{\theta}(x, y)(\theta=a$ or $b$ ) of the two gases. As a consequence of Eq. (17) and of the assumption of a constant Prandtl number of the species $\theta, \mu_{\theta}$ and $\kappa_{\theta}$ depend on $T_{\theta}$ only and are both proportional to $T_{\theta}^{\omega_{\theta}}$. Consequently, from (35), $\psi_{a b}$ depends on the two temperatures $T_{a}$ and $T_{b}$ only. Now, from (33), we see that $\hat{S_{a}^{E}}$ is the product of a constant (not depending on $x$ and $y$ ) by $\psi_{a b}\left(T_{b}-T_{a}\right)\left(p_{a} \rho_{b}\right) / \mu_{a}=\left(T_{b}-T_{a}\right)\left(\psi_{a b} p_{a} p_{b}\right) /\left(\mu_{a} r_{b} T_{b}\right)$. Of course, a similar expression is obtained for $\hat{S_{b}^{E}}$ by exchanging $a$ and $b$.

As a result, the two equations (31) for $\theta=a$ and $b$ are two second-order ordinary differential equations for the two temperatures $T_{a}$ and $T_{b}$, with respect to the variable $y$ and $x$ being a variable parameter. In conclusion, the coefficients present in these differential equations depend only on constants of the problem, the parameter $x$, and the two temperatures $T_{a}$ and $T_{b}$. These equations and the temperature jump boundary conditions (34) on the walls $y= \pm 1 / 2$ are given below in (36) and (37), in which $D_{a}, D_{b}, E_{a}$, and $E_{b}$ are positive quantities and do not depend on $y$

$$
\begin{aligned}
& \frac{\partial}{\partial y}\left(T_{a}^{\omega_{a}} \frac{\partial T_{a}}{\partial y}\right)=D_{a} \frac{1}{T_{a}^{\left(\omega_{a}+1\right) / 2} T_{b}^{\left(\omega_{b}+1\right) / 2}}\left(T_{a}-T_{b}\right), \\
& \frac{\partial}{\partial y}\left(T_{b}^{\omega_{b}} \frac{\partial T_{b}}{\partial y}\right)=D_{b} \frac{1}{T_{a}^{\left(\omega_{a}+1\right) / 2} T_{b}^{\left(\omega_{b}+1\right) / 2}}\left(T_{b}-T_{a}\right),
\end{aligned}
$$

$$
\begin{gathered}
T_{a}=T_{w} \mp E_{a} T_{a}^{\omega_{a}+1 / 2} \frac{\partial T_{a}}{\partial y}, \quad T_{b}=T_{w} \mp E_{b} T_{b}^{\omega_{b}+1 / 2} \frac{\partial T_{b}}{\partial y}, \\
\text { in } y= \pm 1 / 2 .
\end{gathered}
$$

Finding analytical solutions is not obvious. Fortunately, there is an evident solution: $T_{a}(x, y)=T_{b}(x, y)=T_{w}(x)$. If $T_{a}$ and $T_{b}$ take values close to $T_{w}(x)$, the linearized system obtained from Equations (36) and (37) admits only the solution $T_{a}(x$, $y)=T_{b}(x, y)=T_{w}(x)$ (see Appendix C).

Due to the ideal gas laws, $p_{\theta}=\rho_{\theta} T_{\theta}$, the volumetric masses $\rho_{\theta}$ depend on $x$ only. Consequently, the stress viscosity $\mu_{\theta}$ depends on $T_{\theta}$ only, and therefore, $\mu_{\theta}$ is a known function of $x$.

Using the ideal gas law and the expression of $\psi_{a b}$ as given in Eq. (35), the coupling term $\hat{S}_{a}^{u}(32)$ appears as the product of three factors: a positive constant $F_{a b} \sqrt{\frac{\pi}{2 \gamma_{a}}} \frac{M_{a c}}{K n_{a c}} \sqrt{\Omega_{a} \Omega_{b}}$, a known positive function depending on the variable $x$ which is $\left(\mu_{a} \mu_{b}\right)^{-1 / 2}\left(T_{a} T_{b}\right)^{-1 / 2}$, and a third unknown factor $p_{a}(x) p_{b}(x)\left(u_{b}(x, y)-u_{a}(x, y)\right)$.

The dimensionless balance equations for mass (29) and longitudinal momentum (30), and the dimensionless longitudinal slip boundary condition (34) can now be written. For species $a$, we have

$$
\begin{gathered}
\frac{\partial}{\partial x}\left(\frac{p_{a}}{T_{w}} u_{a}\right)+\frac{\partial}{\partial y}\left(\frac{p_{a}}{T_{w}} v_{a}\right)=0, \\
\frac{\partial^{2} u_{a}}{\partial y^{2}}=B_{a}\left(u_{a}-u_{b}\right)+A_{a} \frac{d p_{a}}{d x}, \\
u_{a}=\mp K_{a} \frac{\partial u_{a}}{\partial y}+H_{a} \frac{d T_{w}}{d x} \text { in } y= \pm 1 / 2
\end{gathered}
$$

with

$$
\begin{gathered}
B_{a}=F_{a b} \sqrt{\frac{\pi}{2 \gamma_{a}}}\left(\frac{1}{K n_{a c}}\right)^{2} \Omega_{a} \psi_{a b} \frac{p_{a} p_{b}}{\mu_{a}^{2} T_{b}}, \quad A_{a}=\frac{1}{M_{a c} K n_{a c} \mu_{a}}, \\
K_{a}=\sigma_{a P} \frac{2}{\sqrt{\pi}} K n_{a c} \sqrt{T_{a}} \frac{\mu_{a}}{p_{a}}, \quad H_{a}=\sigma_{a T} \frac{2}{\pi} \frac{K n_{a c}}{M_{a c}} \frac{\mu_{a}}{p_{a}} .
\end{gathered}
$$

For species $b$, the equations are obtained by exchanging $a$ and $b$. The two ordinary differential equations for $u_{a}$ and $u_{b}$ are solved as follows: 


$$
\begin{aligned}
u_{a}= & \Upsilon \frac{y^{2}}{2}-\frac{A_{a}}{\psi^{2}} \frac{d p_{a}}{d x}+C_{1}+C_{2} y \\
& +B_{a}\left[C_{3} \cosh (\psi y)+C_{4} \sinh (\psi y)\right], \\
u_{b}= & \Upsilon \frac{y^{2}}{2}-\frac{A_{b}}{\psi^{2}} \frac{d p_{b}}{d x}+C_{1}+C_{2} y \\
& -B_{b}\left[C_{3} \cosh (\psi y)+C_{4} \sinh (\psi y)\right],
\end{aligned}
$$

where

$$
\psi=\sqrt{B_{a}+B_{b}}, \quad \Upsilon=\frac{B_{b} A_{a}}{\psi^{2}} \frac{d p_{a}}{d x}+\frac{B_{a} A_{b}}{\psi^{2}} \frac{d p_{b}}{d x},
$$

where $C_{1}, C_{2}, C_{3}$, and $C_{4}$ are four integration constants. The physical problem is symmetrical with respect to the $x$-axis. Consequently, $C_{2}=C_{4}=0$. The two last constants, $C_{1}$ and $C_{3}$, are calculated from the boundary conditions (39) written for the species $a$ and $b$. After calculus, we obtain explicit expressions for $C_{1}$ and $C_{3}$ (see Appendix D).

In order to obtain the pressure of each gas, the local balance mass Equation (38) for the two species are integrated from $y=-1 / 2$ to $y=+1 / 2$, with the wall boundary condition for the transversal velocities $v_{a}=v_{b}=0$ at $y= \pm 1 / 2$. Then

$$
\int_{-1 / 2}^{+1 / 2} \frac{p_{a}}{T_{w}} u_{a} d y=Q_{a}, \quad \int_{-1 / 2}^{+1 / 2} \frac{p_{b}}{T_{w}} u_{b} d y=Q_{b},
$$

where $Q_{a}$ and $Q_{b}$ are constants and correspond to the dimensionless mass flow rates of the two species $a$ and $b$. We substitute the expressions (41) for $u_{a}$ and $u_{b}$ into Eq. (43), and we perform the integration with respect to variable $y$,

$$
\begin{aligned}
& \frac{1}{24} \Upsilon+2 B_{a} C_{3} \frac{\sinh (\psi / 2)}{\psi}+C_{1}-\frac{A_{a}}{\psi^{2}} \frac{d p_{a}}{d x}=\frac{T_{w}}{p_{a}} Q_{a}, \\
& \frac{1}{24} \Upsilon-2 B_{b} C_{3} \frac{\sinh (\psi / 2)}{\psi}+C_{1}-\frac{A_{b}}{\psi^{2}} \frac{d p_{b}}{d x}=\frac{T_{w}}{p_{b}} Q_{b} .
\end{aligned}
$$

The quantities $\Upsilon, C_{1}$, and $C_{3}$ are affine expressions in $d p_{a} / d x$ and $d p_{b} / d x$ (see Eq. (46) and Appendix D). After calculus, we have

$$
\mathcal{A}_{a a} \frac{d p_{a}}{d x}+\mathcal{A}_{a b} \frac{d p_{b}}{d x}=\widehat{Q}_{a}, \quad \mathcal{B}_{b a} \frac{d p_{a}}{d x}+\mathcal{B}_{b b} \frac{d p_{b}}{d x}=\widehat{Q}_{b}
$$

The explicit values of $\mathcal{A}_{a a}, \mathcal{A}_{a b}, \mathcal{B}_{b a}, \mathcal{B}_{b b}, \widehat{Q}_{a}$, and $\widehat{Q}_{b}$ are given in Appendix D. These six quantities depend on the constant parameters which characterize the two gases, on the temperature $T_{w}(x)$ of the walls and on the two pressures $p_{a}(x)$ and $p_{b}(x)$. Additionally, the quantity $\widehat{Q}_{a}$ (or $\widehat{Q}_{b}$ ) is an affine function of the dimensionless flow rate $Q_{a}$ (or $Q_{b}$ ), with coefficients depending on the two pressures $p_{a}$ and $p_{b}$ and on known functions of $x$.

We note that the two differential equations (45) are linear with respect to the derivatives $d p_{a}(x) / d x$ and $d p_{b}(x) / d x$, but they are strongly non-linear with respect to the functions $p_{a}(x)$ and $p_{b}(x)$. Indeed, in $\mathcal{A}_{a a}, \mathcal{A}_{a b}, \mathcal{B}_{b a}$, and $\mathcal{B}_{b b}$, there are the functions $\psi, \cosh (\psi / 2)$, and $\sinh (\psi / 2)$ which are non-linear. For example, from Eqs. (40) and (42), we note that $\psi$ is equal to the product of $\sqrt{p_{a} p_{b}}$ by a known function depending on $x$ only.

In order to solve Equation (45) for $x \in[0,1]$, we need four conditions $p_{a}(0), p_{b}(0),\left(d p_{a} / d x\right)(0)$, and $\left(d p_{b} / d x\right)(0)$, for example, or, equivalently, $p_{a}(0), p_{b}(0)$, and the dimensionless flow rates $Q_{a}$ and $Q_{b}$. Indeed from these four last values, the quantities $\widehat{Q}_{a}$ and $\widehat{Q}_{b}$ are known (see Appendix D) and, consequently, the derivatives $d p_{a} / d x(0)$ and $d p_{b} / d x(0)$ are known by Eq. (45). Due to the physics of the problem, $p_{a}(0), p_{b}(0)$, $Q_{a}$, and $Q_{b}$ are four positive quantities. From the theory of ordinary differential equations, there exists a single solution each for $p_{a}(x)$ and $p_{b}(x)$ for positive $x$ and close to $x=0$. For our physical problem, this solution should be such that $p_{a}(x)$ and $p_{b}(x)$ are positive on the entire interval $[0,1]$. As a result, the function $\psi$ should be always defined and have a positive real value. A theoretical study of system (45) has not yet been carried out. Finally, we note that the solutions of Equation (45) are very sensitive to the initial values $p_{a}(0)$ and $p_{b}(0)$ and to the mass flow rates $Q_{a}$ and $Q_{b}$. Depending on the values of these quantities, the pressures $p_{a}(x)$ and $p_{b}(x)$ may have very different behaviors. Note also that, with a bad choice for these quantities, there are no real solutions to Equation (45) in the entire range $0 \leq x \leq 1$.

\section{NUMERICAL SOLUTION}

In order to make appropriate physical choices for the four quantities $p_{a}(0), p_{b}(0), Q_{a}$, and $Q_{b}$, a DSMC simulation has been performed. In a two-dimensional microchannel of length $L=20 \mu \mathrm{m}$ and height $h=1 \mu \mathrm{m}$, we consider the steady flow of two monatomic gases, argon and neon, denoted by $a$ and $b$, respectively. Additionally, the problem is assumed to be isothermal with $T^{*}=300 \mathrm{~K}$, and the interactions between the molecules are described using the variable soft sphere (VSS) model. ${ }^{54}$ The physical characteristics $m_{\theta}, d_{\theta}, \omega_{\theta}, \alpha_{\theta}$, and $\mu_{\theta}^{r e f}$ associated with $T_{\theta}^{r e f}=273 \mathrm{~K}$ are given in Bird's book. ${ }^{54}$ They are recalled in Table I.

The DSMC simulation results will be given in Section VI. Here, only the orders of magnitude of some quantities are given. For the gas pressures at the microchannel inlet, we take $p_{a \text { in }}^{*}=35000 \mathrm{~Pa}$ and $p_{b i n}^{*}=15000 \mathrm{~Pa}$, and at the microchannel outlet, we take $p_{a \text { out }}^{*}=28000 \mathrm{~Pa}$ and $p_{\text {bout }}^{*}$ $=12000 \mathrm{~Pa}$. For the mixture mean speed and for the gas mass

TABLE I. Characteristic values of monatomic gases from Bird's book. ${ }^{54}$

\begin{tabular}{lcccccc}
\hline \hline Gas & Symbol & $\begin{array}{c}\text { Molecular mass } \\
\times 10^{-27}(\mathrm{~kg})\end{array}$ & $\begin{array}{c}\text { Viscosity at 273 K } \\
\times 10^{-5}\left(\mathrm{~N} \mathrm{~s} \mathrm{~m}^{-2}\right)\end{array}$ & $\begin{array}{c}\text { Viscosity } \\
\text { index } \omega\end{array}$ & $\begin{array}{c}\text { VSS parameter } \\
\alpha\end{array}$ & $\begin{array}{c}\text { Molecule diameter } \\
\times 10^{-10}(\mathrm{~m})\end{array}$ \\
\hline Helium & $\mathrm{He}$ & 6.65 & 1.865 & 0.66 & 1.26 & 2.30 \\
Neon & $\mathrm{Ne}$ & 33.5 & 2.975 & 0.66 & 1.31 & 2.72 \\
Argon & $\mathrm{Ar}$ & 66.3 & 2.117 & 0.81 & 1.40 & 4.11 \\
Krypton & $\mathrm{Kr}$ & 139.1 & 2.328 & 0.80 & 1.32 & 4.70 \\
Xenon & $\mathrm{Xe}$ & 218 & 2.107 & 0.85 & 1.44 & 5.65 \\
\hline \hline
\end{tabular}


flow rates, DSMC simulation yields the following approximate values: $U^{*}=5 \mathrm{~m} \mathrm{~s}^{-1}, Q_{a}^{*}=2.25 \times 10^{-6} \mathrm{~kg} \mathrm{~m}^{-1} \mathrm{~s}^{-1}$, and $Q_{b}^{*}=0.49 \times 10^{-6} \mathrm{~kg} \mathrm{~m}^{-1} \mathrm{~s}^{-1}$. Now, we introduce the following characteristic values: $T_{c}=300 \mathrm{~K}, P_{c}=10^{4} \mathrm{~Pa}, U_{c}=10 \mathrm{~m} \mathrm{~s}^{-1}$, $\rho_{a c}=m_{a} P_{c} /\left(k T_{c}\right)=0.16 \mathrm{~kg} \mathrm{~m}^{-3}, \quad \rho_{b c}=m_{b} P_{c} /\left(k T_{c}\right)=0.081$ $\mathrm{kg} \mathrm{m}^{-3}, Q_{a c}=\rho_{a c} U_{c} h=1.60 \times 10^{-6} \mathrm{~kg} \mathrm{~m}^{-1} \mathrm{~s}^{-1}$, and $Q_{b c}$ $=\rho_{b c} U_{c} h=0.81 \times 10^{-6} \mathrm{~kg} \mathrm{~m}^{-1} \mathrm{~s}^{-1}$. Consequently, we have the following dimensionless quantities: $p_{a}^{D S M C}(0)=3.5$, $p_{b}^{D S M C}(0)=1.5, Q_{a}^{D S M C}=Q_{a}^{*} / Q_{a c}=1.41$, and $Q_{b}^{D S M C}=Q_{b}^{*} /$ $Q_{b c}=0.60$.

In order to solve Eq. (45), we take four values for $p_{a}(0)$, $p_{b}(0), Q_{a}$, and $Q_{b}$ around the orders of magnitude of the above values. In all the calculations presented here, the characteristic scales for the temperatures, pressures, and velocities are $T_{c}$ $=300 \mathrm{~K}, P_{c}=10^{4} \mathrm{~Pa}$, and $U_{c}=10 \mathrm{~m} \mathrm{~s}^{-1}$, respectively. The characteristic values for the two gases in the mixture are given in Table I. The microchannel's geometry is $L=20 \mu \mathrm{m}$, $h=1 \mu \mathrm{m}$, and $\varepsilon=0.05$. The characteristic stress viscosity values are the viscosities $\mu_{\theta}^{*}(300 \mathrm{~K})$ calculated with formula (17). Additionally, we set $\sigma_{\theta P}=1$ and $\sigma_{\theta T}=1.1 .{ }^{37}$ Calculations with MATLAB using a variable step Runge-Kutta method of fourth order are performed to determine the pressures $p_{\theta}(x)$. Then the velocities $u_{\theta}(x, y)$ are calculated. The concentration $c_{\theta}$ of the species $\theta$ defined as $c_{\theta}=n_{\theta}^{*} /\left(n_{a}^{*}+n_{b}^{*}\right)=p_{\theta} /\left(p_{a}+p_{b}\right)$ depends on $x$ only and is also calculated.

Three sets of results are shown: (1) the isothermal flow of a mixture of two gases-argon and neon; (2) the isothermal flow of different binary gas mixtures; (3) the non-isothermal flow of a mixture of two gases-argon and neon.

\section{A. Isothermal flow of a mixture of two gases-Argon and neon}

The two simulated gases are argon $(\operatorname{Ar}$, gas $a$ ) and neon $\left(\mathrm{Ne}\right.$, gas $b$ ); the flow is isothermal, with $T_{\theta}^{*}=T_{c}=300 \mathrm{~K}$.

The initial values for the dimensionless pressures are $p_{a}(0)$ $=3.5$ and $p_{b}(0)=1.5$. These values correspond to concentrations $c_{a}(0)=0.7$ and $c_{b}(0)=0.3$. The value of the dimensionless

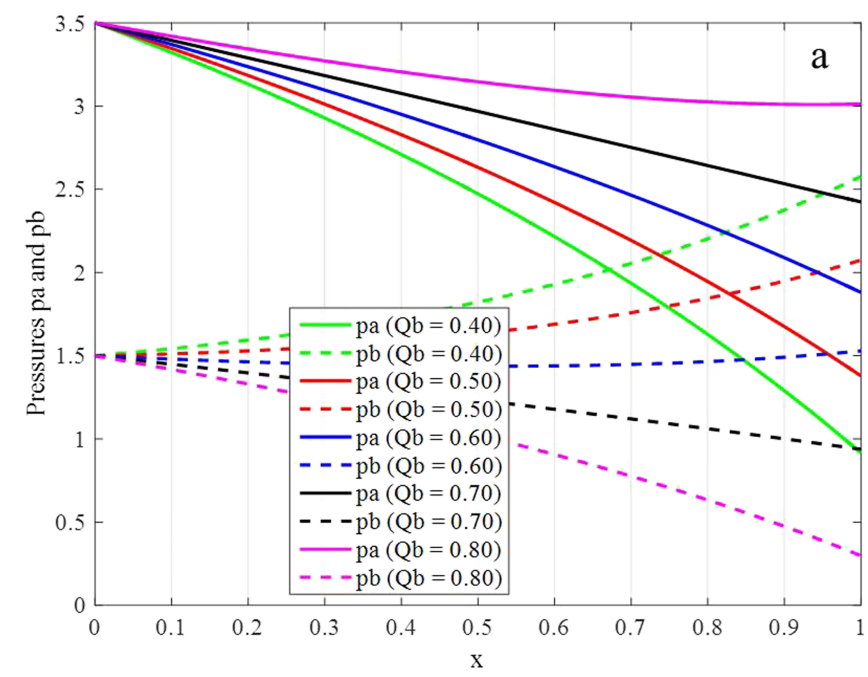

mass flow rate $Q_{a}=1.60$ is given, and the dimensionless mass flow rate $Q_{b}$ takes different values: $0.40,0.50,0.60,0.70$, and 0.80 . For these selected values, the profiles for the two pressures are monotonically increasing or decreasing along the $x$ axis (Fig. 1(a)).

For a fixed value of $x$, when $Q_{b}$ increases from 0.40 to 0.80 , the pressure $p_{a}(x)$ increases and the pressure $p_{b}(x)$ decreases (Fig. 1(a)). For the sake of brevity, the results for the concentration are not presented here, but they are similar to those for pressures: when $\mathrm{x}$ increases, the concentration $c_{a}(x)$ increases and the concentration $c_{b}(x)$ decreases. Of course, $c_{a}(x)+c_{b}(x)$ $=1$.

In Fig. 1(b), the longitudinal velocities along the $x$-axis, $u_{a}(x, 0)$ and $u_{b}(x, 0)$, are shown. For a fixed value of $x$, when $Q_{b}$ increases from 0.40 to 0.70 , the velocity $u_{a}(x, 0)$ decreases and the velocity $u_{b}(x, 0)$ increases. These results are in agreement with those obtained for the pressures. The velocities obtained with $Q_{b}=0.80$ are not shown, in the interest of readability of the figure (with $Q_{b}=0.80$, the value $u_{b}(1,0)=2.73$ is high, compared to the other four cases). For $Q_{b}$ equal to 0.40 to 0.70 , when $x$ increases $u_{a}(x, 0)$ increases. The velocity $u_{b}(x$, 0 ) decreases when $Q_{b}$ is equal to $0.40,0.50$, and 0.60 and increases when $Q_{b}$ is equal to 0.70 .

Of course, when the mass flow rate $Q_{b}$ increases, the total mass flow rate $Q_{a}+Q_{b}$ increases. For a fixed value of $x$, we can show that the increase of $Q_{a}+Q_{b}$ leads to the decrease of the total pressure $p(x)=p_{a}(x)+p_{b}(x)$. Additionally, when $x$ increases from 0 to 1 , the total pressure decreases and the mixture velocity $u(x)=\left(m_{a} u_{a}(x)+m_{b} u_{b}(x)\right) /\left(m_{a}+m_{b}\right)$ increases. This last result seems to be physically reasonable.

Now, the initial values of pressures are unchanged and the flow rates are set as $Q_{a}=1.60$ and $Q_{b}=0.60$. For different values of $x(x=0,0.25,0.50,0.75,1)$, the profiles of the longitudinal velocities $u_{a}(x, y)$ and $u_{b}(x, y)$ are given in Figs. 2(a) and 2(b). They have an almost parabolic shape (see Eq. (41)). Indeed, for example, for $x=0.5$, the numerical values of $B_{a} C_{3} \cosh (\psi y)$ and $-B_{b} C_{3} \cosh (\psi y)$ are, respectively, between -0.104 and -0.079 and -0.075 and -0.057 when $y$ is between 0 and 0.5 . These values are small compared to

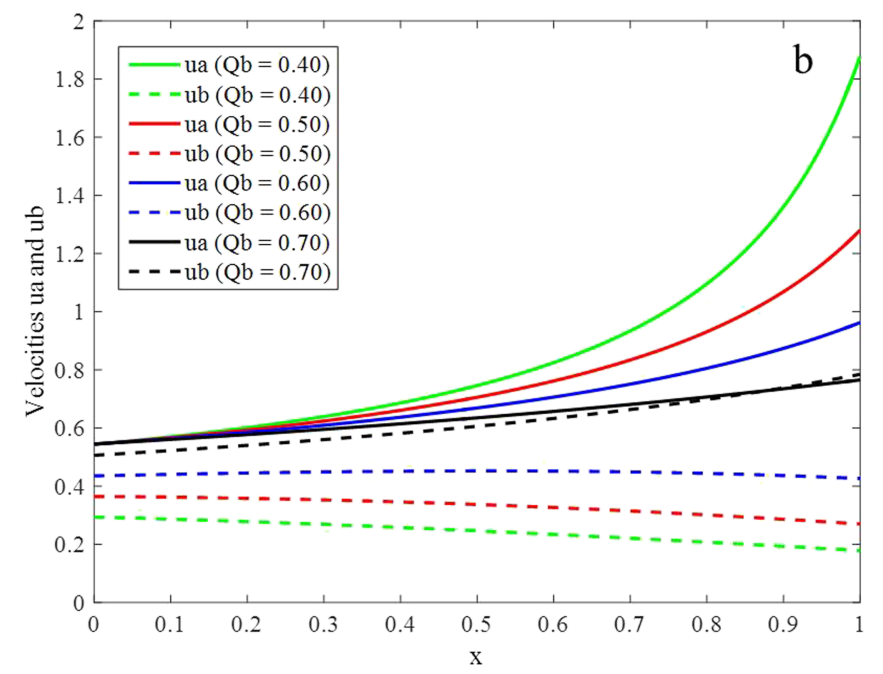

FIG. 1. Pressures $p_{a}(x)$ and $p_{b}(x)$ (a) and velocities $u_{a}(x, 0)$ and $u_{b}(x, 0)$ (b) along the $x$-axis for $p_{a}(0)=3.5, p_{b}(0)=1.5, Q_{a}=1.60$, and different values for $Q_{b}$. Results for the gas $a$ are represented by solid lines, and those of gas $b$ by the dashed lines. 

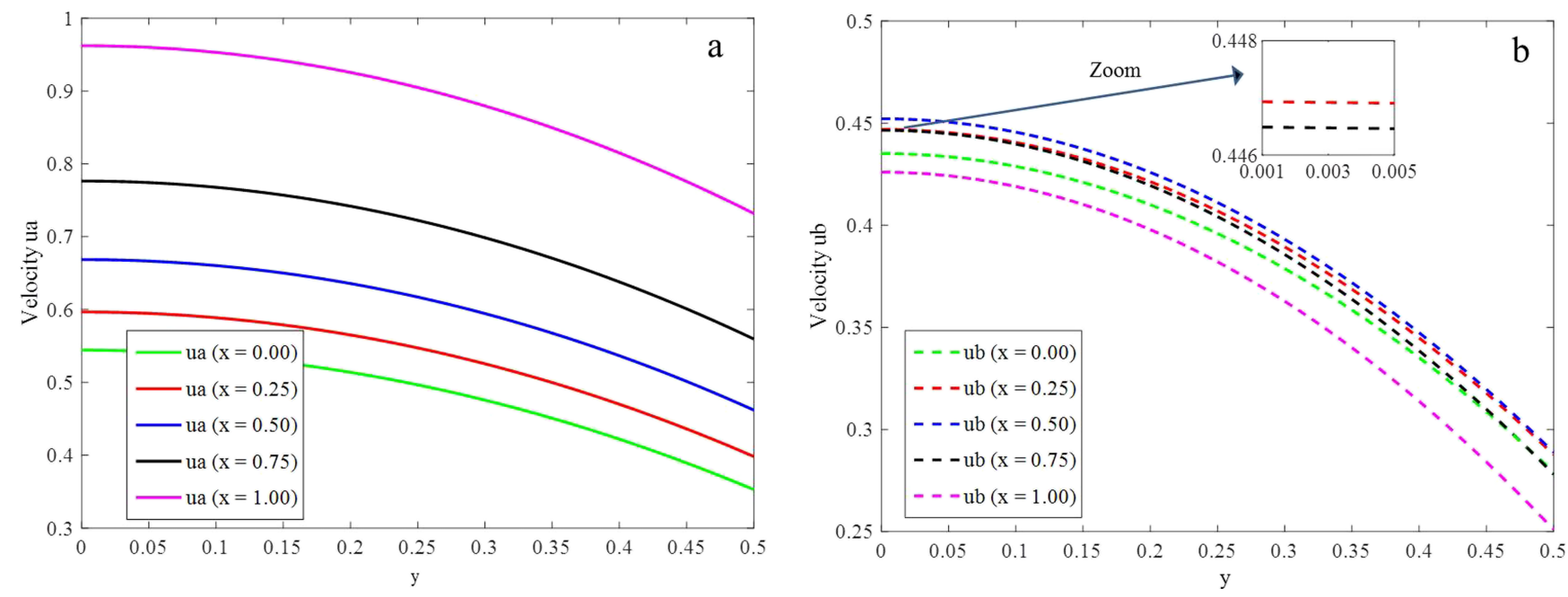

FIG. 2. Velocity profiles for $u_{a}(x, y)$ (a) and $u_{b}(x, y)(\mathrm{b})$, for $p_{a}(0)=3.5, p_{b}(0)=1.5, Q_{a}=1.60, Q_{b}=0.60$, and for different values of $x$.

$C_{1}=0.393$. So the terms $B_{a} C_{3} \cosh (\psi y)$ and $-B_{b} C_{3} \cosh (\psi y)$ are small with respect to $C 1$ and have little influence on the parabolic shape of the profiles.

When $x$ increases, the magnitude of the velocity $u_{a}(x$, $y$ ) increases (Fig. 2(a)). The maximum magnitude in $y=0$ rises from 0.54 to 0.96 . Note also that the slipping velocity $u_{a}(x, \pm 0.5)$ on the walls increases with $x$ from 0.35 to 0.73 . The velocity profiles of the gas with the lowest concentration are close to each other (Fig. 2(b)). It is worthy to note that the maximum magnitude of $u_{b}(x, 0)$ is between 0.43 and 0.45 , and the slipping velocity $u_{b}(x, \pm 0.5)$ is between 0.25 and 0.29 . The slipping velocity of neon is less than that of argon.

Calculations performed with the same values $p_{a}(0)=3.5$, $P b(0)=1.5$, and $Q_{a}=1.60$ and different values for $Q_{b}$ lead to some interesting results in the middle section of the channel $(x=0.5)$. When $Q_{b}$ increases from 0.40 to 0.80 , the slipping velocity for argon $u_{a}(0.5, \pm 0.5)$ and also the maximum velocity $u_{a}(0.5,0)$ obtained on the axis of the channel decrease. For gas $b$ (neon), when $Q_{b}$ increases, the slipping velocity $u_{b}(0.5, \pm 0.5)$ and the maximum velocity $u_{b}(0.5,0)$ increase. Therefore, increasing $Q_{b}$ decreases the velocity of

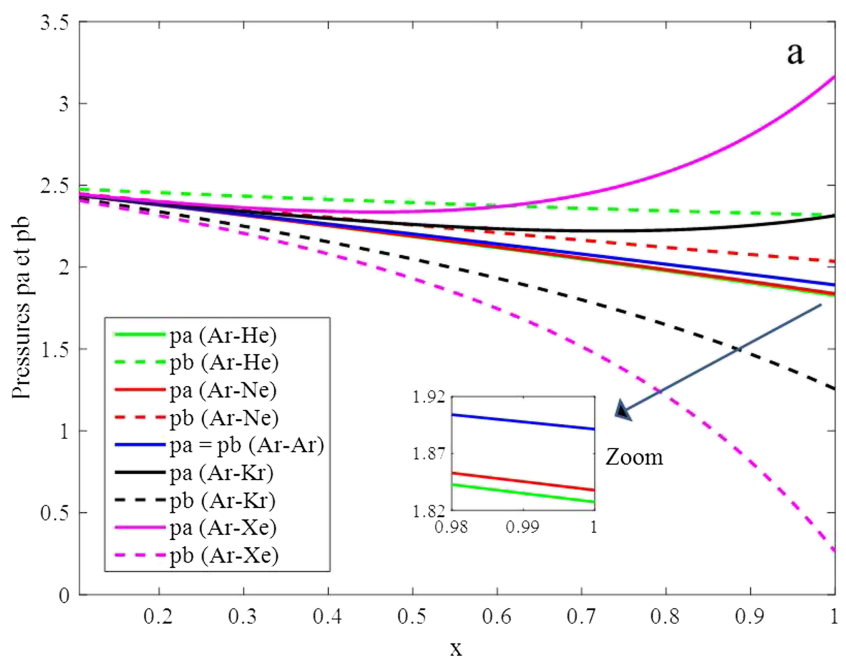

gas $a$ and increases that of gas $b$. These results are physically reasonable.

Now, let us comment the values of $\psi_{a b}^{*}$ and $\psi_{b a}^{*}$ given in Eq. (20). Calculations performed with $Q_{a}=1.60, Q_{b}=0.60$, $p_{a}(0)=3.5$, and $p_{b}(0)=1.5$ give the following results: $p_{a}(1)$ $=1.8802$ and $p_{b}(1)=1.5288$. With $\psi_{a b}^{*}=1$ and $\psi_{b a}^{*}=\beta_{a}^{*} / \beta_{b}^{*}$, which are the values considered in her thesis by Reyhanian, ${ }^{55}$ the relation (19) is satisfied, but these two terms have no symmetrical expressions with respect to the two gases. In that case, the results are $p_{a}(1)=1.8893$ and $p_{b}(1)=1.5202$. Additional tests were made with $\psi_{a b}^{*}=\psi_{b a}^{*}=1$ and with $\psi_{b a}^{*}=1$ and $\psi_{a b}^{*}=\beta_{b}^{*} / \beta_{a}^{*}$. Comparing the final three results with the one corresponding to former choice (20), the relative errors on $p_{a}(1)$ and $p_{b}(1)$ are not more than $0.5 \%$ and $0.6 \%$, respectively. We can consider that these relative errors are small.

\section{B. Isothermal flow of different binary gas mixtures}

In Figs. 3 and 4, different pairs of monatomic gases are considered: gas $a$ is argon ( $\mathrm{Ar}$ ) and gas $b$ is helium (He), neon $(\mathrm{Ne})$, argon $(\mathrm{Ar})$, krypton $(\mathrm{Kr})$, or xenon $(\mathrm{Xe})$.

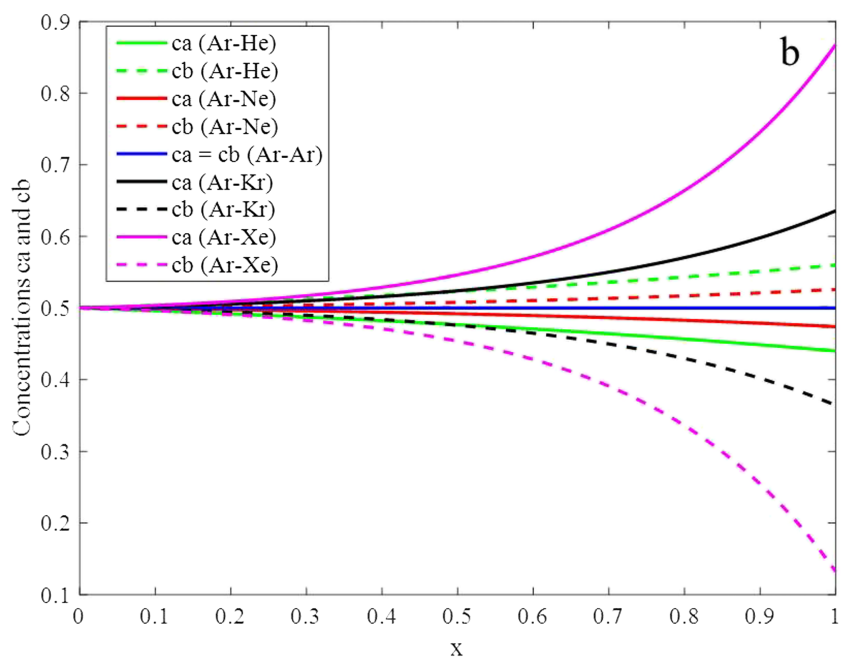

FIG. 3. Pressures (a) and concentrations (b) along the $x$-axis for $p_{a}(0)=p_{b}(0)=2.5$ and $Q_{a}=Q_{b}=0.80$ and for different pairs of gases. 

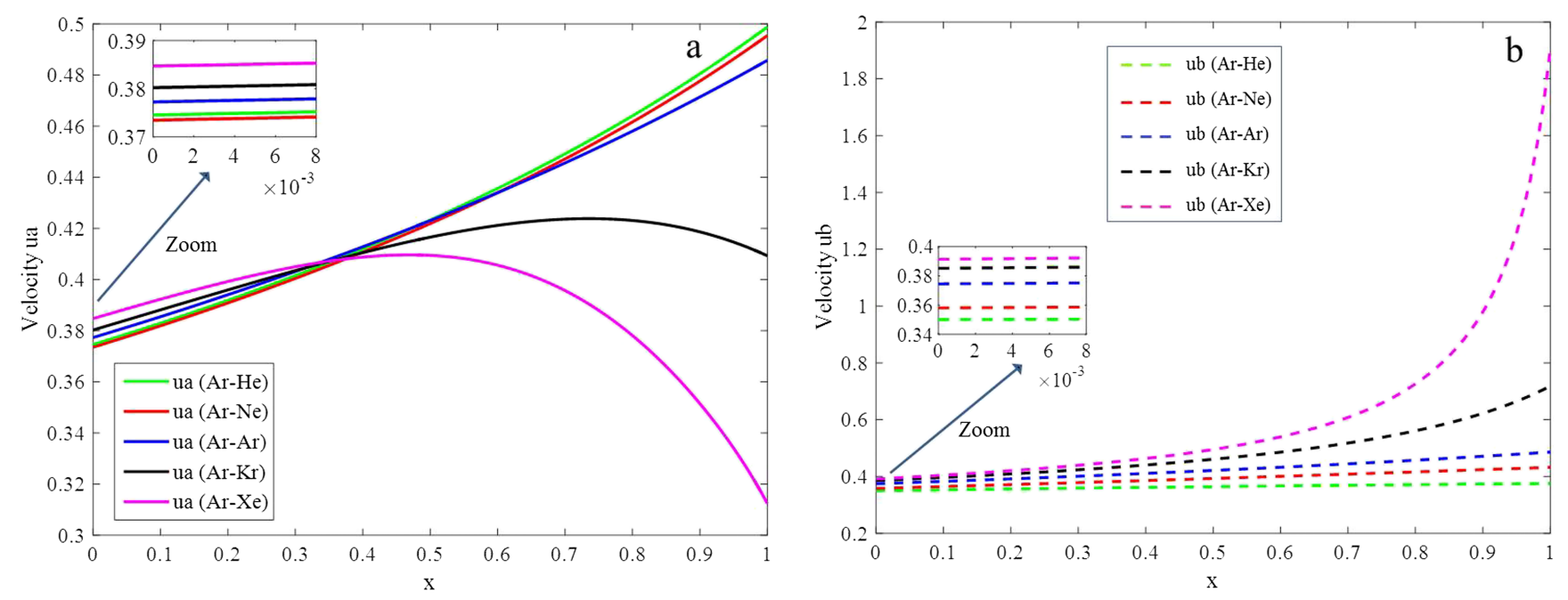

FIG. 4. Velocity $u_{a}$ (a) and velocity $u_{b}$ (b) along the $x$-axis for $p_{a}(0)=p_{b}(0)=2.5$ and $Q_{a}=Q_{b}=0.80$ and for different pairs of gases.

The initial values for the pressures are taken to be equal to $p_{a}(0)=p_{b}(0)=2.5$ and the mass flow rates equal to $Q_{a}$ $=Q_{b}=0.80$. It should be noted that the molecular mass increases from helium gas to xenon gas (Table I). For a fixed value of $x$, when the molecular mass increases from $\mathrm{He}$ to $\mathrm{Xe}$, the pressure $p_{a}$ increases and the pressure $p_{b}$ decreases (Fig. 3(a)). For all pairs, the pressure $p_{b}(x)$ decreases along the $x$-axis when the variable $x$ increases. Except for the pairs $(\mathrm{Ar}-\mathrm{Xe})$ and $(\mathrm{Ar}-\mathrm{Kr})$, the pressure $p_{a}(x)$ also decreases with the variable $x$ (Fig. 3(a)). Of course, for Ar-Ar mixture, we have $p_{a}(x)=p_{b}(x)$; this common value decreases with the $x$-coordinate.

The physical characteristics of the gases are different and lead to different behaviors. For a given binary gas and for a fixed value of $x$, we note that the pressure of the gas with the higher molecular mass is smaller than that of the gas with the smaller molecular mass (Fig. 3(a)): $p_{A r}(x)<p_{\mathrm{He}}(x)$, $p_{A r}(x)<p_{N e}(x), p_{A r}(x)>p_{K r}(x)$, and $p_{A r}(x)>p_{X e}(x)$. In a collision between two molecules with different molecular masses, the lighter molecule has a larger change in its speed than the heavier molecule; this increases its momentum and, therefore, the pressure.

Now, let us look at the results for the concentrations $c_{a}(x)$ and $c_{b}(x)$ (Fig. 3(b)). For a fixed value of $x$, when the molecular mass increases from $\mathrm{He}$ to $\mathrm{Xe}$, the concentration $c_{a}(x)$ increases and the concentration $c_{b}(x)$ decreases. Of course, for the mixture $\operatorname{Ar}-\operatorname{Ar}, c_{a}(x)=c_{b}(x)=0.5$. Along the $x$ axis, when the variable $x$ is increasing, the concentration $c_{a}(x)$ (respectively, $c_{b}(x)$ ) is monotonically increasing (respectively decreasing) for the mixtures $\mathrm{Ar}-\mathrm{Xe}$ and $\mathrm{Ar}-\mathrm{Kr}$ and is monotonically decreasing (respectively increasing) for the mixtures $\mathrm{Ar}-\mathrm{Ne}$ and $\mathrm{Ar}-\mathrm{He}$. The concentration of the gas with the larger molecular mass is decreasing whereas that of the gas with the lower molecular mass is increasing. This property holds for all binary mixtures of two gases taken among the five selected monoatomic gases.

For the velocities $u_{a}(x, 0)$ and $u_{b}(x, 0)$, the analysis of the results is a little more complicated (Figs. 4(a) and 4(b)). Near the microchannel exit, when the molecular mass of species $b$ increases (from He to $\mathrm{Xe}$ ), $u_{a}(1,0)$ is decreasing (Fig. 4(a)) and $u_{b}(1,0)$ is increasing (Fig. 4(b)). However near the microchannel entrance, the velocity $u_{a}(0,0)$ has no uniform behavior (Fig. 4(a)). For a given binary mixture and for a fixed value of $y$, we can verify that, in the middle section of the channel, the velocity of the gas with the higher molecular mass is higher than that of the gas with the smaller molecular mass. That is, $u_{A r}>u_{H e}, u_{A r}>u_{N e}, u_{A r}<u_{K r}$, and $u_{A r}<u_{X e}$ at the point $(x=0.5, y)$, i.e., in the entire interval $-0.5 \leq y \leq 0.5$. Of course, the same inequalities are taking place for the velocity maxima $u_{a}(0.5,0)$ and $u_{b}(0.5,0)$ and also for the slip velocities on the walls $u_{a}(0.5, \pm 0.5)$ and $u_{b}(0.5, \pm 0.5)$. This is physically reasonable.

\section{Non-isothermal flow of a mixture of two gases-Argon and neon}

Let us consider the previous geometry for the microchannel and the two gases, $\operatorname{argon}(a)$ and neon $(b)$. Here, we impose the same temperature $T_{w}^{*}\left(x^{*}\right)=T_{c} T_{w}(x)$ along the two channel walls. Solving Equation (45) is performed by a linear variation of $T_{w}(x): T_{w}(x)=1+(d T / d x) x$ with $d T / d x$ constant. Hereafter, the selected values for $d T / d x$ are $-0.4,-0.2,0.0,0.2$, and 0.4 . A first set (case I) of results is shown with the flow rates such as $Q_{a}=Q_{b}=0.80$ and the initial pressures such as $p_{a}(0)$ $=p_{b}(0)=2.5$. A second set (case II) of results is shown with the flow rates such as $Q_{a}=1.60, Q_{b}=0.60, p_{a}(0)=3.5$, and $p_{b}(0)=1.5$. In both cases, for the flows of the two gases $a$ and $b$, there is competition between the effects due to the pressure gradients and those due to the temperature gradients.

In case I, the pressure evolution along the $x$-axis is shown (Fig. 5(a)). For the values of $d T / d x$ between -0.2 and 0.2 , the two pressures $p_{a}(x)$ and $p_{b}(x)$ are monotonically decreasing with $x$. One can also observe that, with $d T / d x=0.4$, the pressure $p_{b}(x)$ has a non-monotonic behavior, and with $d T / d x=-0.4$, it is the pressure $p_{a}(x)$ which has a non-monotonic behavior. For a fixed value of $x$, when $d T / d x$ increases, we observe that the pressure $p_{a}(x)$ decreases and the pressure $p_{b}(x)$ increases. An increase of temperature on the walls decreases the pressure $p_{a}(x)$ of argon, which has the highest molecular mass, and increases the pressure $p_{b}(x)$ of neon, which has the smallest molecular mass. In other words, due to the boundary conditions 

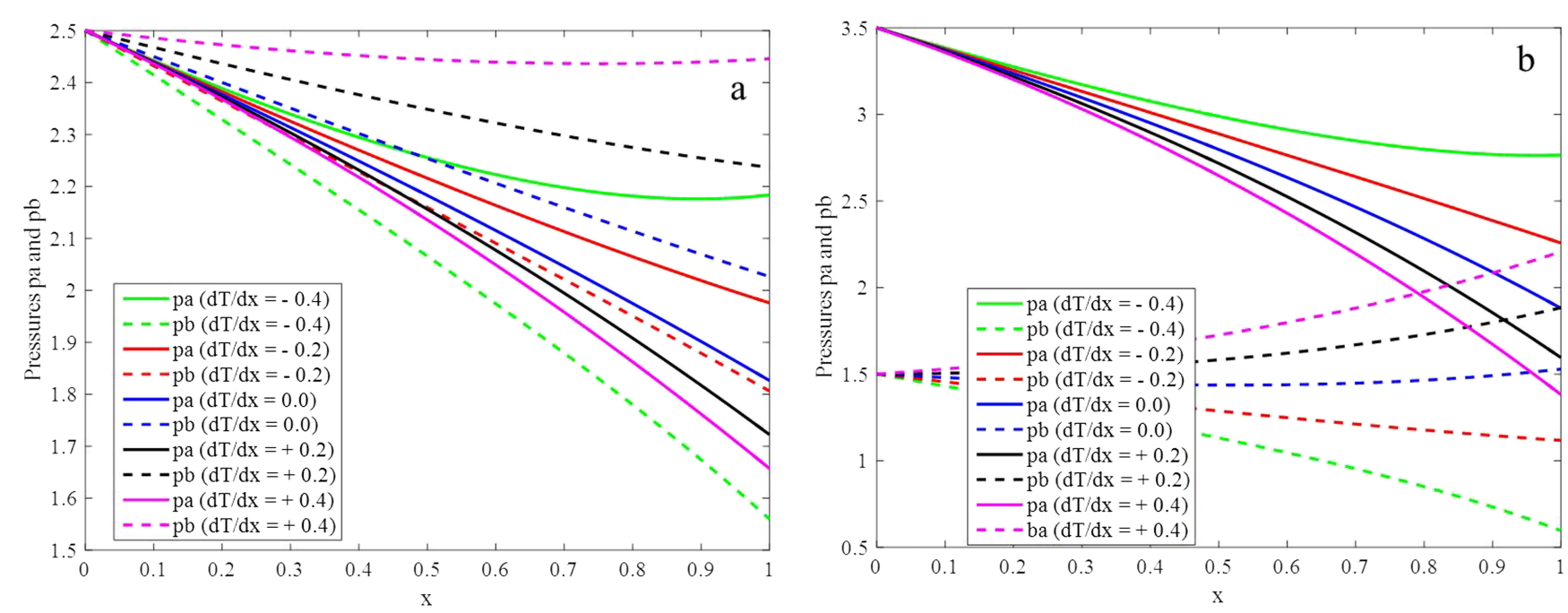

FIG. 5. Pressures for different values of $d T / d x$ along the $x$-axis, for $p_{a}(0)=p_{b}(0)=2.5$ and $Q_{a}=Q_{b}=0.80$ (a) and for $p_{a}(0)=3.5, p_{b}(0)=1.5, Q_{a}=1.60$, and $Q_{b}=0.60$ (b).

on the walls, the pressure of lighter species increases while that of heavier species decreases.

The velocities $u_{a}(x, y)$ and $u_{b}(x, y)$ were also calculated. Among the obtained results, we give some remarks on the longitudinal velocity profiles $u_{a}(0.5, y)$ and $u_{b}(0.5, y)$ in the middle section of the channel $(x=0.5)$. These profiles are almost parabolic (Eq. (41)). For a fixed value of $y$, when the temperature gradient increases, the value of $u_{a}(0.5, y)$ increases and the value of $u_{b}(0.5, y)$ decreases. With the five selected values of $d T / d x$, the maximum velocity magnitude of $u_{a}(0.5$, $0)$ increases from 0.16 to 0.21 and that of $u_{b}(0.5,0)$ decreases from 0.20 to 0.12 . Additionally, when the temperature gradient increases, the slipping velocity on the walls $u_{a}(0.5, \pm 0.5)$ of gas $a$ increases from 0.09 to 0.16 , and the slipping velocity $u_{b}(0.5, \pm 0.5)$ of gas $b$ decreases from 0.13 to 0.08 .

In case II, similar results are obtained with the same temperature gradients along the walls (Fig. 5(b)). The pressure in gas $a$, which has the greater initial pressure, is monotonically decreasing, except when $d T / d x=-0.4$, while the pressure in gas $b$ is monotonically decreasing if $d T / d x=-0.4$ or -0.2 and is monotonically increasing for the two values $d T / d x=0.4$ or 0.2 and is a non-monotonic function for $d T / d x=0$ (Fig. 5(b)). As previously described, when $d T / d x$ increases, for a fixed value of $x$, the pressure $p_{a}(x)$ decreases and the pressure $p_{b}(x)$ increases. As in case I, the two velocities $u_{a}(x, y)$ and $u_{b}(x, y)$ were calculated. As previously described, in the cross section in the middle of the channel, when the temperature gradient increases, on the one hand, the velocity maximum $u_{a}(0.5,0)$ and the slip velocity $u_{a}(0.5, \pm 0.5)$ of gas $a$ increase, while on the other hand, the velocity maximum $u_{b}(0.5,0)$ and the slip velocity $u_{b}(0.5, \pm 0.5)$ of gas $b$ decrease.

One last comment concerns the total pressure $p(x)$ $=p_{a}(x)+p_{b}(x)$ and the mixture velocity $u(x)=\left(m_{a} u_{a}(x)\right.$ $\left.+m_{b} u_{b}(x)\right) /\left(m_{a}+m_{b}\right)$. In Fig. 6, they are shown in the case I. For a fixed value of $x$, the increase of the gas temperature leads to the increase of the pressure $p(x)$ (Fig. 6(a)). The two functions $p_{1}(x)$ and $p_{2}(x)$ associated with $(d T / d x)_{1}<(d T / d x)_{2}$ are such that $p_{1}(x)<p_{2}(x)$. This result seems to be physically correct. Whatever be the value of $d T / d x$ greater than $-0.4(d T / d x>-0.4)$, the velocity $u(x)$ increases along the
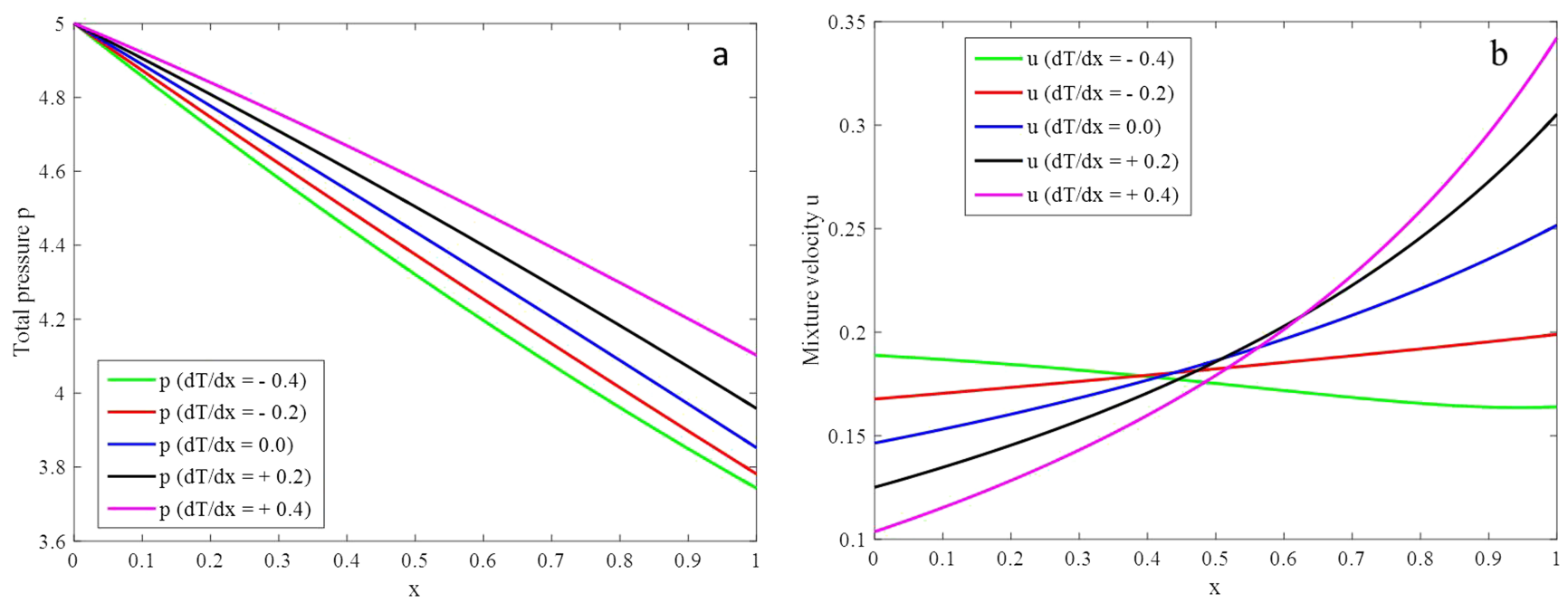

FIG. 6. Total pressure $p(x)$ (a) and average velocity of the mixture $u(x, 0)$ (b) along the $x$-axis for $p_{a}(0)=p_{b}(0)=2.5$ and $Q_{a}=Q_{b}=0.80$ and for different values of $d T / d x$. 
$x$-axis (Fig. 6(b)). Finally, it should be noted that $u(0,0)$ decreases with $d T / d x$, while $u(1,0)$ increases. The velocity profiles $u(x)$ along the $x$-axis intersect (Fig. 6(b)). In the second study (case II), the results are very similar.

As a remark, let us assume that the flows in the microchannel are such that $d p_{a}(x) / d x=d p_{b}(x) / d x=0$. From (41), and the expressions of the constants $C_{1}$ and $C_{3}$ given in Appendix D, we have

$$
\begin{aligned}
u_{a}(x, \pm 0.5)= & \frac{1}{D}\left\{H_{a} \psi^{2} \cosh (\psi / 2)\right. \\
& \left.+\left(B_{b} K_{b} H_{a}+B_{a} K_{a} H_{b}\right) \psi \sinh (\psi / 2)\right\} \frac{d T_{w}}{d x}, \\
u_{b}(x, \pm 0.5)= & \frac{1}{D}\left\{H_{b} \psi^{2} \cosh (\psi / 2)\right. \\
& \left.+\left(B_{a} K_{a} H_{b}+B_{b} K_{b} H_{a}\right) \psi \sinh (\psi / 2)\right\} \frac{d T_{w}}{d x} .
\end{aligned}
$$

It is easy to calculate these velocities with the data of the two cases I and II; the results are, respectively, $u_{a}(x, \pm 0.5)$ $=0.23 d T_{w} / d x, u_{b}(x, \pm 0.5)=0.32 d T_{w} / d x, u(x, \pm 0.5)=0.26$ $d T_{w} / d x$, and $u_{a}(x, \pm 0.5)=0.22 d T_{w} / d x, u_{b}(x, \pm 0.5)=0.38$ $d T_{w} / d x$, and $u(x, \pm 0.5)=0.28 d T_{w} / d x$. When $d T_{w} / d x$ is negative, zero, or positive, the slipping velocities of the two gases $a$ and $b$ and of the mixture is negative, zero, or positive. Also, the three velocities $u_{a}(x, y), u_{b}(x, y)$, and $u(x, y)$ are negative, zero, or positive at any point $(\mathrm{x}, \mathrm{y})$ of the channel. A temperature gradient, negative, zero, or positive, gives velocities $u_{a}$, $u_{b}$, and $u$, negative, zero or positive, respectively. The thermal creep phenomenon, which is well-known in the case of a single rarefied gas, ${ }^{58,59}$ is clear.

\section{COMPARISON BETWEEN ASYMPTOTIC MODEL SOLUTION AND DSMC SIMULATION}

In this section, the aim is to compare, for a flow in a microchannel, the results given by the DSMC simulation and by the asymptotic model. Argon (gas $a$ ) and neon (gas $b$ ) are considered. Of course, the physical characteristics of the two gases are given in Table I and are used both in DSMC simulations and in asymptotic models. The DS2V code of Bird ${ }^{45}$ is used in its two dimensional steady version. The microchannel has length $L=20 \mu \mathrm{m}$ and width $h=1 \mu \mathrm{m}$ and is located between an inlet chamber and an outlet chamber, as in the Ref. 14. The axes $\left(O, x^{*}, y^{*}\right)$ are specified at the beginning of Section II. The walls are at rest and they have the same temperature: $T_{w}^{*}\left(x^{*}\right)$. Additionally, diffuse boundary conditions are assumed with full accommodation to the wall temperature. At the simulation beginning, the gas mixture is at rest and is separated in two parts in the two areas $x^{*}<L / 2$ and $x^{*}>L / 2$. In the both areas, the mixture proportion is $70 \%$ argon and $30 \%$ neon. In the first area, the total pressure is $p_{i n}^{*}=5 \times 10^{4} \mathrm{~Pa}$ and the mixture temperature is $T_{i n}^{*}=300 \mathrm{~K}$; in the second area, the total pressure is $p_{\text {out }}^{*}=4 \times 10^{4} \mathrm{~Pa}$ and the mixture temperature is $T_{\text {out }}^{*}=300 \mathrm{~K}, 305 \mathrm{~K}, 310 \mathrm{~K}, 320 \mathrm{~K}$, or $400 \mathrm{~K}$. Due to the pressure and temperature gradients, the two gases flow through the microchannel. We have five simulations which are, respectively, denoted by Expt. 1, Expt. 2, Expt. 3, Expt. 4, and Expt. 5 (Tables II and III).

In order to compare these DSMC simulation results with the asymptotic results, dimensionless variables are introduced with the characteristic quantities previously defined $\left(x=\left(x^{*} / L, y=y^{*} / h, p_{a}=p_{a}^{*} / P=p_{a}^{*} / 10^{4}\right.\right.$, etc. $)$. As previously described (Section $\mathrm{V}$ ), the characteristic values for the mass flow rates are $Q_{a c}=1.60 \times 10^{-6} \mathrm{~kg} \mathrm{~m}^{-1} \mathrm{~s}^{-1}$ and $Q_{b c}$ $=0.81 \times 10^{-6} \mathrm{~kg} \mathrm{~m}^{-1} \mathrm{~s}^{-1}$. The DSMC simulations give the values of the physical quantities in each Monte Carlo sampling cell. Note that there are about 5100 cells inside the microchannel $(0<x *<L)$, about 245 cells along the longitudinal axis, and 20 cells along a transverse direction. Consequently, the dimensionless pressures $p_{a}^{D S M C}(x, y)$ and $p_{b}^{D S M C}(x, y)$, and the dimensionless velocities $u_{a}^{D S M C}(x, y)$ and $u_{b}^{D S M C}(x, y)$, are known in each cell. Now, we have to calculate the pressures $p_{\theta}^{\text {Model }}(x)(\theta=a, b)$, given by the solutions of the equation set (45). To do this, we have to introduce initial values for the two pressures and values for the two flow rates $Q_{a}^{\text {Model }}$ and $Q_{b}^{\text {Model }}$. In order to make the most meaningful comparison possible, we take the $p_{\theta}^{\text {Model }}(0)$ and $Q_{\theta}^{\text {Model }}$ values in agreement with the DSMC simulation values. More precisely, we consider the results of DSMC simulations. The average values of $p_{a}^{*}\left(x^{*}, y^{*}\right)$ and $p_{b}^{*}\left(x^{*}, y^{*}\right)$ for $y^{*}=0$ and for $x^{*}$ between $0 \mu \mathrm{m}$ and $0.1 \mu \mathrm{m}$ are calculated and are denoted by $P \times p_{a}^{D S M C}(0)$ and $P \times p_{b}^{D S M C}(0)$. These average values are computed using about 30 cells. Similarly, average values $P \times p_{a}^{D S M C}(1)$ and $P \times p_{b}^{D S M C}(1)$ at the channel exit are calculated by taking average values of $p_{a}^{*}\left(x^{*}, y^{*}\right)$ and $p_{b}^{*}\left(x^{*}, y^{*}\right)$ from about 20 cells with $y^{*}=0$ and $x^{*}$ between $19.9 \mu \mathrm{m}$ and $20 \mu \mathrm{m}$. It remains to determine the mass flow rates of the two gas components through the channel. The code DS2V used in our simulations gives the number of molecules $N^{*}$ crossing the line $x^{*}=10 \mu \mathrm{m}$ per second. The average concentration of each mixture component $\theta$ near the channel section $x^{*}=10 \mu \mathrm{m}$ is obtained by taking

TABLE II. DSMC simulations are initialized with $T_{\text {in }}^{*}=300 \mathrm{~K}, p_{\text {a }}^{*}=35000 \mathrm{~Pa}, p_{b \text { in }}^{*}=15000 \mathrm{~Pa}, p_{\text {a out }}^{*}$ $=28000 \mathrm{~Pa}, p_{b \text { in }}^{*}=12000 \mathrm{~Pa}$, and with different $T_{\text {out }}^{*}$ values. DSMC results are given for average Mach number $M a$, average Knudsen number $K n$, the number of molecules $N^{*}$ crossing the line $x^{*}=10 \mu \mathrm{m}$, and for the dimension and dimensionless mass flow rates.

\begin{tabular}{lcccccccc}
\hline \hline $\begin{array}{l}\text { DSMC } \\
\text { simulation }\end{array}$ & $\begin{array}{c}T_{\text {out }}^{*} \\
(\mathrm{~K})\end{array}$ & $\begin{array}{c}\text { Average } \\
M a\end{array}$ & $\begin{array}{c}\text { Average } \\
K n\end{array}$ & $\begin{array}{c}N^{*} \\
\times 10^{-19}\end{array}$ & $\begin{array}{c}Q_{a}^{*} \times 10^{+6} \\
\left(\mathrm{~kg} \mathrm{~m}^{-1} \mathrm{~s}^{-1}\right)\end{array}$ & $\begin{array}{c}Q_{b}^{*} \times 10^{+6} \\
\left(\mathrm{~kg} \mathrm{~m}^{-1} \mathrm{~s}^{-1}\right)\end{array}$ & $Q_{a}^{D S M C}$ & $Q_{b}^{D S M C}$ \\
\hline Expt. 1 & 300 & 0.015 & 0.162 & 2.42 & 2.248 & 0.485 & 1.405 & 0.600 \\
Expt. 2 & 305 & 0.016 & 0.163 & 2.50 & 2.322 & 0.502 & 1.451 & 0.620 \\
Expt. 3 & 310 & 0.017 & 0.165 & 2.62 & 2.440 & 0.523 & 1.525 & 0.646 \\
Expt. 4 & 320 & 0.018 & 0.165 & 2.79 & 2.590 & 0.561 & 1.618 & 0.693 \\
Expt. 5 & 400 & 0.026 & 0.190 & 4.14 & 3.875 & 0.816 & 2.421 & 1.008 \\
\hline \hline
\end{tabular}


TABLE III. Dimensionless pressures of the gases at the microchannel exit $(x=1)$ given by the DSMC simulations and the asymptotic results calculated with $Q_{\theta}^{M o d e l}=Q_{\theta}^{D S M C}$ and $p_{\theta}^{\text {Model }}(0)=p_{\theta}^{D S M C}(0)(\theta=a$ and $b)$. In the last two columns, relative errors on the pressures $p_{a}(1)$ and $p_{b}(1)$ are given.

\begin{tabular}{lccccccccc}
\hline \hline $\begin{array}{l}\text { DSMC } \\
\text { simulation }\end{array}$ & $\begin{array}{c}\left(T_{\text {in }}^{*}-T_{\text {out }}^{*}\right) \\
/ T_{c}\end{array}$ & $\begin{array}{c}p_{a}^{D S M C} \\
(0)\end{array}$ & $\begin{array}{c}p_{b}^{\text {DSMC }} \\
(0)\end{array}$ & $\begin{array}{c}p_{a}^{D S M C} \\
(1)\end{array}$ & $\begin{array}{c}p_{b}^{\text {DSMC }} \\
(1)\end{array}$ & $\begin{array}{c}p_{a}^{\text {Model }} \\
(1)\end{array}$ & $\begin{array}{c}p_{b}^{\text {Model }} \\
(1)\end{array}$ & $\begin{array}{c}\text { Error } \\
\text { on } p_{a}(1)(\%)\end{array}$ & $\begin{array}{c}\text { Error } \\
\text { on } p_{b}(1)(\%)\end{array}$ \\
\hline Expt. 1 & 0 & 3.5331 & 1.5142 & 2.7613 & 1.1773 & 2.5239 & 1.1074 & 8.6 & 5.9 \\
Expt. 2 & 0.01667 & 3.5625 & 1.5220 & 2.7521 & 1.1832 & 2.5046 & 1.1229 & 9.0 & 5.1 \\
Expt. 3 & 0.03333 & 3.5869 & 1.5358 & 2.7482 & 1.1788 & 2.4067 & 1.1903 & 12.4 & 1.0 \\
Expt. 4 & 0.06667 & 3.6491 & 1.5563 & 2.7420 & 1.1781 & 2.3965 & 1.1955 & 12.6 & 1.5 \\
Expt. 5 & 0.33333 & 4.0797 & 1.7152 & 2.6563 & 1.1424 & 1.3769 & 1.9810 & 48.2 & 73.4 \\
\hline \hline
\end{tabular}

the average value of $c_{\theta}^{*}\left(x^{*}, y^{*}\right)$ from about 50 cells centred on $x^{*}$-axis and located in the interval $[9.9 \mu \mathrm{m}, 1.1 \mu \mathrm{m}]$. From this average concentration number, the molecular mass of the molecule $\theta$, and the number $N^{\star}$ defined previously, it is easy to calculate the dimension mass flow rate $Q_{\theta}^{*}=Q_{\theta c}$ $\times Q_{\theta}^{D S M C}$.

Quantities $Q_{\theta}^{D S M C}, p_{\theta}^{D S M C}(0)$, and $p_{\theta}^{D S M C}(1)$ are calculated for the five DSMC simulations; they are given in Tables II and III.

There is a large amount of statistical noise in DSMC calculations $;^{54,55}$ therefore, these values are somewhat approximate. More specifically, relative errors in the initial values of the pressures are about $0.1 .^{60}$ Errors on the mass flow rates are much higher. This is due to the fact that the Mach number, on order of $10^{-2}$, is small in the five simulations (Table II). According to the paper of Hadjiconstantinou, ${ }^{60}$ the error on the velocities is inversely proportional to the Mach number. As a result, the velocity error is approximately 100 times larger than the pressure error. From this remark, we can assume that the error of the mass flow rate is about $10 \%$.

Associated with each simulation, Expt. 1 to Expt. 5, the mass flow rates $Q_{a}^{\text {Model }}=Q_{a}^{D S M C}$ and $Q_{b}^{\text {Model }}=Q_{b}^{D S M C}$ and the initial values of pressures $p_{a}^{\text {Model }}(0)=p_{a}^{D S M C}(0)$ and $p_{b}^{\text {Model }}(0)=p_{b}^{D S M C}(0)$ are introduced. Then, with these four quantities, by using MATLAB, the ordinary differential equations (45) are solved. Additionally, the viscous and thermal slip coefficients are those of full accommodation with the walls $\left(\sigma_{\theta P}=\sigma_{\theta T}=1\right)$. The solutions $p_{a}^{\text {model }}(x)$ and $p_{b}^{\text {model }}(x)$ of (45) are calculated, and the obtained values $p_{a}^{\text {Model }}(1)$ and $p_{b}^{\text {Model }}(1)$ are set in Table III. In the two last rows of this table, the relative errors on the pressures at the channel exit defined by $\left|\left(p_{\theta}^{D S M C}(1)-p_{\theta}^{\text {Model }}(1)\right) / p_{\theta}^{D S M C}(1)\right|$ are given.

For the four experiments, Expt. 1 to Expt. 4, the relative difference is, at most, $13 \%$ for argon and $6 \%$ for neon. These errors have the same order of magnitude as the errors on the values $p_{\theta}^{D S M C}(0)$ and $Q_{\theta}^{D S M C}$ which are used to solve the equation set (45). The relative error of the pressure of argon is much greater than that of the pressure of neon. Is it a result of the argon concentration being greater than that of neon or that the molecular mass of argon is greater than that of neon? These questions are not fully understood and answered. Moreover, the sum of both relative errors of the two pressures is always around 14\%. For Expt. 5, the results at exit of the microchannel are very disappointing. The relative errors on the pressures are $48 \%$ with argon and $73 \%$ for neon. In that case, it should be noted that the ratio $\left(T_{\text {out }}^{*}-T_{\text {in }}^{*}\right) / T_{c}=100 / 300=0.33$ is relatively large. This can be a cause of non-agreement between the DSMC simulation and asymptotic solution.

Other simulations were performed and have led to similar results. For example, let us consider five new DSMC simulations for the binary mixture of argon and neon, in the previous microchannel. As previously described, in the inlet chamber, we have $p_{i n}^{*}=50000 \mathrm{~Pa}$ and $T_{i n}^{*}=300 \mathrm{~K}$, and in the outlet chamber, $p_{\text {out }}^{*}=40000 \mathrm{~Pa}$ and $T_{\text {out }}^{*}=300,305,310,320$, or $400 \mathrm{~K}$. Additionally, we have the same linear temperature profiles on both walls and equal percentages for argon and neon at the beginning of each simulation. The relative errors $\left|\left(p_{\theta}^{D S M C}(1)-p_{\theta}^{\text {Model }}(1)\right) / p_{\theta}^{D S M C}(1)\right|$ with $\theta=a$ and $b$ have respective values $6 \%, 20 \%, 21 \%, 26 \%, 69 \%$ and $13 \%, 2 \%$, $1 \%, 4 \%, 36 \%$ when the temperature of the mixture in the outlet chamber increases from $300 \mathrm{~K}$ to $400 \mathrm{~K}$. It is noted that the previous remarks on the relative errors for experiments Expt. 1 to Expt. 5 can be repeated here.

Now, we compare the pressure profiles along the microchannel's $x$-axis given by the DSMC simulation and the asymptotic model. Two sets of results are shown: first, with the wall temperature equal to a constant (Expt. 1) and second with the wall temperature increasing from $300 \mathrm{~K}$ to $320 \mathrm{~K}$ (Expt. 4). The profiles $p_{\theta}^{\text {Model }}(x)(\theta=a, b)$ correspond to the solutions of Equation (45). For the DSMC results, we are interested in the values of the pressures on the $x$-axis, $p_{\theta}^{D S M C}(x, 0)$ $(\theta=a, b)$, denoted simply by $p_{\theta}^{D S M C}(x)$. In other words, we take the pressure values in DSMC cells centered at $y=0$. The dimensionless pressure profiles are presented as solid lines in the case of asymptotic solutions and with markers in the case of DSMC results (Figs. 7(a) and 7(b)). For both experiments, the pressure profiles for neon are very close, in contrast to those for argon. The relative errors fluctuate and coarsely increase with the longitudinal abscisse, the error being much higher for argon compared with that for neon. Of course, near the channel outlet $(x=1)$, they agree to the values given in Table III. We note also the quasi-linear evolution of the pressures along the $x$-axis.

Finally, we give the velocity profiles along the channel axis, $u_{\theta}^{\text {Model }}(x, 0)$ and $u_{\theta}^{D S M C}(x, 0)$, for both experiments: Expt. 1 and Expt. 4. For the asymptotic model, $u_{\theta}^{\text {Model }}(x, 0)$ is calculated from Eq. (41) and the values of the two pressures $p_{a}^{\text {Model }}(x)$ and $p_{b}^{\text {Model }}(x)$. From the DSMC simulation results, $u_{\theta}^{D S M C}(x, 0)$ is built by using the same method as that used to build $p_{\theta}^{D S M C}(x)$. The profiles are shown in Figs. 8(a) and 8(b). We must note that the DSMC solutions are very noisy, with large fluctuations, for argon and for neon. 

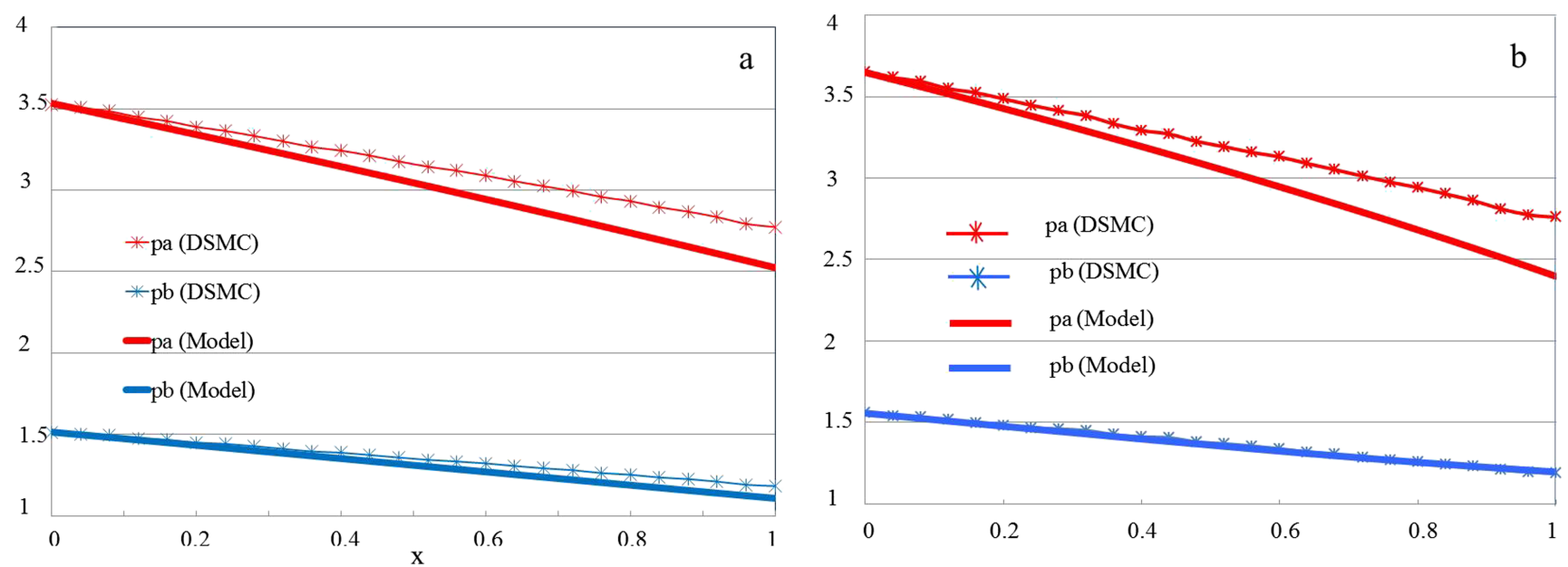

FIG. 7. Dimensionless pressure profiles along the $x$-axis given by the DSMC simulations and by the asymptotic model, in the cases of Expt. 1 (a) and Expt. 4 (b).
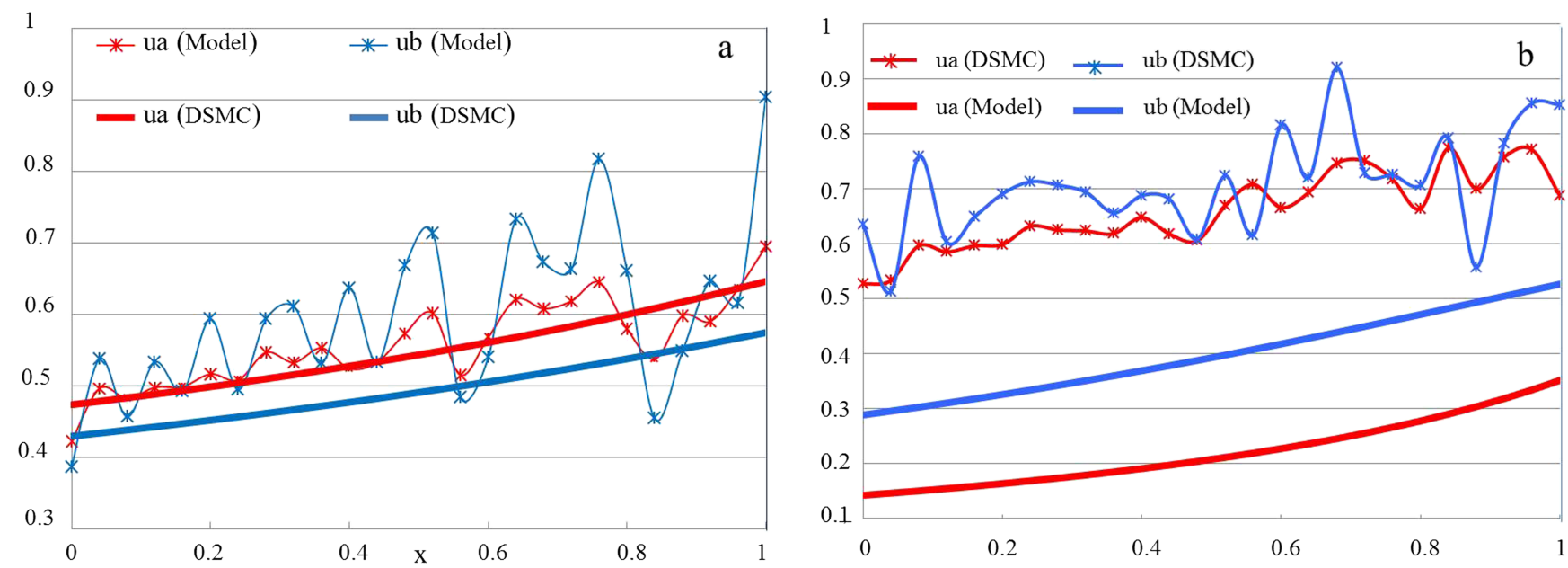

FIG. 8. Dimensionless velocities along the $x$-axis given by the DSMC simulation and by the asymptotic model, in the cases of Expt. 1 (a) and Expt. 4 (b).

In the case of Expt. 1, fluctuations defined | $\left(u_{\theta}^{D S M C}(x, 0)\right.$ $\left.-u_{\theta}^{\text {Model }}(x, 0)\right) / u_{\theta}^{D S M C}(x, 0) \mid$ have the maximum values $13 \%$ for gas $a$ and $36 \%$ for gas $b$, and they have average values 5\% for gas $a$ and $18 \%$ for gas $b$. In the case of Expt. 4, the maximum values are $75 \%$ for gas $a$ and $60 \%$ for gas $b$, and the average values are $67 \%$ for gas $a$ and $44 \%$ for gas $b$. For Expt. 1, it can be concluded that the agreement is roughly correct; however for Expt. 4, the agreement is bad. Due to these fluctuations, the agreement between the two profiles, asymptotic and DSMC, is not very good. Only roughly increasing character of the DSMC profiles is consistent with the asymptotic models. In both experiments Expt. 1 and Expt. 4, the Mach numbers are weak being around $2 \times 10^{-2}$. Other comparisons are planned to be performed with higher Mach numbers on the order of $\varepsilon=0.05$, with the hope of seeing smaller velocity fluctuations.

\section{CONCLUSION}

An asymptotic model has been presented for the flow of a thermal binary gas mixture in a microchannel. It is constructed from the Navier-Stokes-Fourier balance equations with first-order boundary conditions for the velocities and temperatures. The pressures and longitudinal velocities along the symmetry axis of the micro-channel are studied. The results are related to the influence of the mass flow rates, to the comparison between different species pairs, and to the influence of a temperature gradient along the microchannel walls.

Many results were obtained over a given range of data. For instance, for the isothermal Ar-Ne mixture in the proportions $70 \%$ and $30 \%$, respectively, the mass flow rate of argon being fixed, it was shown that, for a fixed transverse section of the microchannel, when the mass flow rate of neon increases, the pressure of argon increases and the pressure of neon decreases. It was also shown that the slipping velocity on the walls of neon is less than that of argon.

In the case of isothermal flows of two monatomic gas mixtures, with the same flow rates and the same concentrations, it is shown that the pressure in the gas with the largest molecular mass is smaller than that with the smallest molecular mass. This could be explained by the remark that in a collision between two molecules, the lightest molecule has a larger change in its speed than the heaviest molecule. For slipping velocities on the walls, it is also shown that the velocity for the lightest gas is smaller than that for the heaviest gas.

In the case of non-isothermal flow mixtures of argon and neon, with the same flow rates and the same concentrations, 
the results were as follows: for a fixed transverse section of the microchannel, the pressure in the gas with the heaviest molecular mass decreases as the temperature gradient increases. On the contrary, the pressure in the lightest gas increases. These results are due to the boundary conditions on the walls. Same remarks can be made in the second study with the $\mathrm{Ar}-\mathrm{Ne}$ mixture in the proportion $70 \%$ argon and $30 \%$ neon, and the nondimensionless flow rates equal to 1.60 and 0.60 , respectively. On both walls that have a non-uniform temperature, the boundary conditions contribute to flow velocities proportional to the temperature gradient in addition to the slip velocities due to viscosity. This is the thermal creep well known in the case of a single rarefied gas. In the present paper, the slipping velocities of the two gases are due to the pressure gradients and the temperature gradient. Both effects are mixed. To study the thermal creep only, an asymptotic model without pressure gradients is planned.

Several comparisons between DSMC simulations and corresponding asymptotic solutions for $\mathrm{Ar}-\mathrm{Ne}$ mixture were made. First, we were interested in pressure profiles along the $x$-axis. For concentrations of $70 \%$ argon and $30 \%$ neon, and a low temperature gradient along the walls, the two solutions are in fairly good agreement. With equal concentrations for both gases, the results are similar. It was noted that the agreement between the two solutions is better with the lightest gas. It was also noted that the results are disappointing with a large temperature gradient. Second, we were interested in velocity profiles. In the isothermal case, the agreement for the velocities of the two gases given by the DSMC simulation and asymptotic model is not too bad; we have not the same conclusion in the non-isothermal case with a temperature gradient of 20/300. Further studies are to be conducted.

Finally, it is important to note that obtaining an asymptotic solution is very fast (less than $1 \mathrm{~s}$ ) compared to obtaining a DSMC result (several hours on a PC). In the future, we need to complete this study by comparing more flows based on DSMC simulations with the corresponding asymptotic solutions. The goal is to better understand the disagreements and to specify the domain of validity of the asymptotic model. In the case of polyatomic molecules, the asymptotic model presented in Refs. 55-57 will eventually be improved and then tested on various mixtures.

\section{NOMENCLATURE}

\begin{tabular}{|c|c|}
\hline Exposant $*$ & $=$ Dimension quantities \\
\hline Indice $c$ & $=$ Characteristic quantities \\
\hline Indices $a, b, \theta$ & $=$ Gas species \\
\hline$v_{\theta}^{*}$ & $=$ Self-collision frequency \\
\hline$v_{a b}^{*}, v_{b a}^{*}$ & $\begin{aligned}= & \text { Cross-collision frequency between } \\
& \text { molecules } a \text { and } b\end{aligned}$ \\
\hline$S_{\theta}^{\mathbf{u} *}, S_{\theta}^{E *}$ & $\begin{array}{l}=\text { Coupling terms in momentum and } \\
\text { energy balance equations }\end{array}$ \\
\hline$S_{\theta}^{u}, S_{\theta}^{v}, S_{\theta}^{E}$ & $\begin{aligned}= & \text { Coupling terms in dimensionless } \\
& \text { momentum and energy equations }\end{aligned}$ \\
\hline$\alpha_{\theta}$ & $\begin{array}{l}=\text { Variable soft sphere (VSS) parame- } \\
\text { ter }\end{array}$ \\
\hline
\end{tabular}

$$
\begin{array}{ll}
\omega_{\theta} & =\text { Viscosity index } \\
\psi_{a b}^{*},\left(\psi_{a b}\right)_{c}, \psi_{a b}, F_{a b}= & \text { Quantities present in the coupling } \\
& \text { terms } \\
\beta_{a b}^{*}, \beta_{b a}^{*} & \text { Quantities present in } \psi_{a b}^{*} \text { and } \psi_{b a}^{*} \\
\sigma_{\theta P}, \sigma_{\theta T}, \zeta_{\theta T} & =\text { Viscous slip, thermal slip, jump } \\
& \text { temperature coefficients on the } \\
& \text { walls }
\end{array}
$$

\section{APPENDIX A: BGK-TYPE EQUATIONS FOR BINARY MIXTURE OF POLYATOMIC GASES}

Most real gases, under standard temperature conditions, satisfy with a very good approximation, the model of perfect gas with constant specific heats. The energy of such a gas is the sum of the energies of each molecule. The energy of a molecule is the sum of the translational kinetic energy of the molecule and different internal energy (see Landau and Lifchitz, Chap. $4^{61}$ and Rahimi and Struchtrup ${ }^{48}$ ). We introduce the temperature $\mathrm{T}$ of the gas defined from the translational energy.

Let us consider a polyatomic gas in thermal equilibrium, under standard conditions of temperature and pressure. The energy of a molecule is the sum of the translational and rotational energies, the other degrees of freedom are frozen. In relation to the equipartition principle of energy, it is established that $c_{v}=\ell k i t / 2$ and $c_{p}=(\ell+2) k i t / 2$, where $\ell=6$ for a nonlinear molecule and $\ell=5$ for a linear molecule. ${ }^{61}$ Hence, $\gamma=c_{p} / c_{v}=(\ell+2) / \ell$.

The two kinetic equations (1) and (2) are assumed valid. In order to have the energy conservation for the mixture, the Morse formulas (5) and (6) for the temperatures must be modified. We propose the following expressions for the pseudo-temperatures $\bar{T}_{a}^{*}$ and $\bar{T}_{b}^{*}$ :

$$
\bar{T}_{a}^{*}=T_{a}^{*}+3\left(\gamma_{a}-1\right) \frac{m_{a} m_{b}}{\left(m_{a}+m_{b}\right)^{2}}\left(T_{b}^{*}-T_{a}^{*}+\frac{m_{b}}{6 k}\left(\mathbf{u}_{b}^{*}-\mathbf{u}_{a}^{*}\right)^{2}\right),
$$

$$
\bar{T}_{b}^{*}=T_{b}^{*}+3\left(\gamma_{b}-1\right) \frac{m_{a} m_{b}}{\left(m_{a}+m_{b}\right)^{2}}\left(T_{a}^{*}-T_{b}^{*}+\frac{m_{a}}{6 k}\left(\mathbf{u}_{a}^{*}-\mathbf{u}_{b}^{*}\right)^{2}\right) .
$$

For monatomic gases, these new expressions are identical to those given in Eqs. (5) and (6). In the balance energy equation (10), only the expression of the coupling term needs to be changed. For the two gases $a$ and $b$, they are

$S_{a}^{E^{*}}=v_{a b}^{*} \rho_{a}^{*}\left[\frac{r_{a}}{\gamma_{a}-1}\left(\bar{T}_{a}^{*}-T_{a}^{*}\right)+\frac{1}{2}\left(\overline{\mathbf{u}}_{a}^{* 2}-\mathbf{u}_{a}^{* 2}\right)\right]$,
$S_{b}^{E^{*}}=v_{b a}^{*} \rho_{b}^{*}\left[\frac{r_{b}}{\gamma_{b}-1}\left(\bar{T}_{b}^{*}-T_{b}^{*}\right)+\frac{1}{2}\left(\overline{\mathbf{u}}_{b}^{* 2}-\mathbf{u}_{b}^{* 2}\right)\right]$.

It is easy to see that the coupling terms $S_{a}^{E^{*}}$ and $S_{b}^{E^{*}}$ have a zero sum for all gases.

\section{APPENDIX B: PRINCIPLE OF LEAST DEGENERACY APPLIED TO EQS. (23)-(25)}

We assume $M_{\theta}=O\left(\varepsilon^{\alpha}\right)$ and $K n_{\theta}=O\left(\varepsilon^{\beta}\right)$ with $\alpha$ $\geq \beta \geq 0$. It is easy to give, a priori, the different orders 
of magnitude of the terms that are present in Eqs. (23)-(25). They are, respectively, $\varepsilon^{2 \alpha}, \varepsilon^{0}, \varepsilon^{\alpha+\beta+1}, \varepsilon^{\alpha+\beta-1}$, and, $\varepsilon^{\alpha-\beta-1}$, the last magnitude order corresponding to the right member of Eq. (23); $\varepsilon^{2 \alpha}, \varepsilon^{-2}, \varepsilon^{\alpha+\beta-1}, \varepsilon^{\alpha+\beta+1}$, and, $\varepsilon^{\alpha-\beta-1}$, where the latter term corresponds to the right-hand side of Eq. (24); $\varepsilon^{0}, \varepsilon^{\beta-\alpha+1}, \varepsilon^{\beta-\alpha-1}, \varepsilon^{\alpha+\beta+1}, \varepsilon^{\alpha+\beta-1}, \varepsilon^{\alpha+\beta+3}$, and, $\varepsilon^{-\alpha-\beta-1}, \varepsilon^{\alpha-\beta-1}, \varepsilon^{\alpha-\beta+1}$, where the last three orders of magnitude correspond to the right-hand side of Eq. (25). Analysis of the magnitudes of the terms listed above shows that only the following orders of magnitude are, respectively, retained: $\varepsilon^{2 \alpha}, \varepsilon^{0}, \varepsilon^{\alpha+\beta-1}$, and $\varepsilon^{\alpha-\beta-1}$ in Eq. (23); $\varepsilon^{-2}$ in Eq. (24); $\varepsilon^{\beta-\alpha-1}, \varepsilon^{\alpha+\beta-1}$, and $\varepsilon^{-\alpha-\beta-1}, \varepsilon^{\alpha-\beta-1}$ in Eq. (25).

Due to the principle of least degeneracy, ${ }^{53}$ we must keep the greatest possible number of terms in Eqs. (23) and (25). Retaining four terms in Eq. (23) is not possible. It is easy to see that there are four possibilities to select three terms in Eq. (23). They are $\alpha=0$ and $\beta=-1 ; \alpha=-1$ and $\beta=0 ; \alpha=0$ and $\beta=+1 ; \alpha=1$ and $\beta=0$. The first two options are not possible. The third possibility is to be rejected as Eqs. (23) and (25) are reduced to only coupling terms. The fourth possibility is feasible with $\alpha=1$ and $\beta=0$.

\section{APPENDIX C: A REMARK ON THE TEMPERATURES $T_{a}(x, y)$ AND $T_{b}(x, y)$}

In the first-order solution (Section IV), the two temperatures $T_{a}(x, y)$ and $T_{b}(x, y)$ are solutions of the two differential equations (36) and satisfy the boundary conditions (37).
We recall that the quantities $D_{a}, D_{b}, E_{a}$, and $E_{b}$ are positive quantities and do not depend on $y$. Now, we assume that $T_{\theta}(x, y)$ takes values close to $T_{w}(x)$. This means $T_{\theta}(x, y)$ $=T_{w}(x)\left(1+t_{\theta}(x, y)\right)$ with $t_{\theta}(x, y)$ very small compared to 1 $\left(t_{\theta}(x, y) \ll 1\right)$. Let us introduce $t_{\theta}(x, y)$ in Eqs. (36) and (37). After linearization, we get:

$\frac{\partial^{2} t_{a}(x, y)}{\partial y^{2}}=e_{a}(x)\left(t_{a}-t_{b}\right), \quad \frac{\partial^{2} t_{b}(x, y)}{\partial y^{2}}=e_{b}(x)\left(t_{b}-t_{a}\right)$,

$t_{a}(x, y)=-\epsilon \varphi_{a}(x) \frac{\partial t_{a}}{\partial y}, \quad t_{b}(x, y)=-\epsilon \varphi_{b}(x) \frac{\partial t_{b}}{\partial y}$,

in $y=\epsilon 1 / 2$

with $\epsilon= \pm 1$. In Eqs. (C1) and (C2), the quantities $e_{a}(x), e_{b}(x)$, $\varphi_{a}(x)$, and $\varphi_{a}(x)$ are known positive functions depending only on the variable $x$. The solutions of the second order ordinary differential equations $(\mathrm{C} 1)$ follow

$t_{\theta}=\frac{\eta e_{\theta}}{e_{a}+e_{b}}\left(K \cosh \left(\sqrt{e_{a}+e_{b}} y\right)+H \sinh \left(\sqrt{e_{a}+e_{b}} y\right)\right)+L y+M$

with $\eta=+1$ for the species $a$, and $\eta=-1$ for the species $b$, where $K, H, L$, and $M$ are four integration constants, which are determined from the boundary conditions (C2),

$$
\begin{aligned}
& \frac{\eta e_{\theta}}{e_{a}+e_{b}}\left(K \cosh \left(\sqrt{e_{a}+e_{b}} / 2\right)+\epsilon H \sinh \left(\sqrt{e_{a}+e_{b}} / 2\right)\right)+\epsilon L / 2+M \\
& \quad=-\epsilon \varphi_{\theta}\left[\frac{\eta e_{\theta}}{\sqrt{e_{a}+e_{b}}}\left(\epsilon K \sinh \left(\sqrt{e_{a}+e_{b}} / 2\right)+H \cosh \left(\sqrt{e_{a}+e_{b}} / 2\right)\right)+L\right] .
\end{aligned}
$$

The relation $(\mathrm{C} 4)$ yields four different equations corresponding to the parameters $(\theta, \eta, \epsilon)$ equal to $(a,+1,+1),(a,+1,-1)$, $(b,-1,+1)$, and $(b,-1,-1)$. We deduce two homogeneous linear systems of equations, of the one part for $K$ and $M$, and on the other part for $L$ and $H$, whose determinant is different of zero.
Accordingly, $K=M=0$ and $L=K=0$. In conclusion, Eqs. (C1) and $(\mathrm{C} 2)$ have the following solutions: $t_{a}(x, y)=t_{b}(x, y)=0$. This corresponds to $T_{a}(x, y)=T_{b}(x, y)=T_{w}(x)$. In other words, if the values of $T_{a}(x, y)$ and $T_{b}(x, y)$ are assumed close to $T_{w}(x)$, the only possible solutions are $T_{a}(x, y)=T_{b}(x, y)=T_{w}(x)$.

\section{APPENDIX D: EXPRESSIONS OF $c_{1}, c_{3}, \mathcal{A}_{a a}, \mathcal{A}_{a b}, \mathcal{B}_{b a}, \mathcal{B}_{b b}, \widehat{Q}_{a}$ AND $\widehat{Q}_{b}$}

First, the expression of $F_{a b}$ deduced from Eqs. (27) and (35) is written as follows:

$$
F_{a b}=\sqrt{\frac{\pi \gamma_{a}}{2}} \frac{\left(d_{a}+d_{b}\right)^{2}}{4 d_{a} d_{b}} \frac{\rho_{b c}}{\rho_{a c}}\left(\frac{\mu_{a c}}{\mu_{b c}}\right)^{1 / 2}\left(\frac{m_{b}}{m_{a}}\right)^{1 / 4}\left(\frac{m_{a}^{2}}{2 m_{b}\left(m_{a}+m_{b}\right)}\right)^{1 / 2},
$$

and the expression of $B_{a}$ deduced from Eqs. (40) and (35) is also written as follows:

$$
B_{a}=F_{a b} \sqrt{\frac{\pi}{2 \gamma_{a}}}\left(\frac{1}{K n_{a c}}\right)^{1 / 2}\left(\frac{\Omega_{a} \Omega_{b} \mu_{a}}{T_{a} T_{b} \mu_{b}}\right)^{1 / 2} p_{a} p_{b} .
$$

The expressions of $A_{a}, K_{a}$, and $H_{a}$ are given in Eqs. (40). Here, they are recalled

$$
A_{a}=\frac{1}{M_{a c} K n_{a c} \mu_{a}}, \quad K_{a}=\sigma_{a P} \frac{2}{\sqrt{\pi}} K n_{a c} \sqrt{T_{a}} \frac{\mu_{a}}{p_{a}}, \quad H_{a}=\sigma_{a T} \frac{2}{\pi} \frac{K n_{a c}}{M_{a c}} \frac{\mu_{a}}{p_{a}} .
$$


Of course, expressions of $F_{b a}, B_{b}, A_{b}, K_{b}$, and $H_{b}$ are obtained from those of $F_{a b}, B_{a}, A_{a}, K_{a}$, and $H_{a}$ by exchanging the species $a$ and $b$.

Second, as in Eq. (42), we set

$$
\psi=\sqrt{B_{a}+B_{b}}, \quad \Upsilon=\frac{B_{b} A_{a}}{\psi^{2}} \frac{d p_{a}}{d x}+\frac{B_{a} A_{b}}{\psi^{2}} \frac{d p_{b}}{d x},
$$

and then, we introduce

$$
D=\psi^{2} \cosh (\psi / 2)+\left(B_{a} K_{a}+B_{b} K_{b}\right) \psi \sinh (\psi / 2)
$$

We have

$$
\begin{aligned}
C_{3}= & \frac{1}{D}\left\{\frac{1}{\psi^{2}}\left(A_{a} \frac{d p_{a}}{d x}-A_{b} \frac{d p_{b}}{d x}\right)-\frac{1}{2} \Upsilon\left(K_{a}-K_{b}\right)+\left(H_{a}-H_{b}\right) \frac{d T_{w}}{d x}\right\}, \\
C_{1}= & -\frac{\Upsilon}{8}+\frac{1}{D}\left\{\frac{\Upsilon}{2}\left[\left(2-B_{b} K_{a}-B_{a} K_{b}\right) \cosh (\psi / 2)-K_{a} K_{b} \psi^{3} \sinh (\psi / 2)\right]\right. \\
+ & \left(B_{b} K_{b} A_{a} \frac{d p_{a}}{d x}+B_{a} K_{a} A_{b} \frac{d p_{b}}{d x}\right) \frac{\sinh (\psi / 2)}{\psi}+\left(B_{b} H_{a}+B_{a} H_{b}\right) \frac{d T_{w}}{d x} \cosh (\psi / 2) \\
+ & \left.\left(B_{b} H_{a} K_{b}+B_{a} H_{b} K_{a}\right) \frac{d T_{w}}{d x} \psi \sinh (\psi / 2)\right\}, \\
& \mathcal{A}_{a a}=\frac{A_{a}}{\psi^{2} D}\left\{-B_{b} \frac{D}{12}+2 B_{a} \frac{\sinh (\psi / 2)}{\psi}+B_{a} B_{b}\left(K_{b}-K_{a}\right) \frac{\sinh (\psi / 2)}{\psi}\right. \\
& \left.-\frac{1}{2} B_{b}\left(B_{b} K_{a}+B_{a} K_{b}\right) \cosh (\psi / 2)-\frac{1}{2} B_{b} K_{a} K_{b} \psi^{3} \sinh (\psi / 2)\right\}, \\
\mathcal{A}_{a b}= & \frac{B_{a} A_{b}}{\psi^{2} D}\left\{-\frac{D}{12}+\cosh (\psi / 2)-2 \frac{\sinh (\psi / 2)}{\psi}+B_{a}\left(K_{b}-K_{a}\right) \frac{\sinh (\psi / 2)}{\psi}\right. \\
& \left.+K_{a} \psi \sinh (\psi / 2)-\frac{1}{2}\left(B_{b} K_{a}+B_{a} K_{b}\right) \cosh (\psi / 2)-\frac{1}{2} K_{a} K_{b} \psi^{3} \sinh (\psi / 2)\right\}, \\
\widehat{Q}_{a}= & \frac{T_{w}}{p_{a} Q_{a} D}-\frac{d T_{w}}{d x}\left\{2 B_{a}\left(H_{a}-H_{b}\right) \frac{\sinh (\psi / 2)}{\psi}+\left(B_{b} H_{a}+B_{a} H_{b}\right) \cosh (\psi / 2)\right\} \\
& +\left\{\frac{d T_{w}}{d x}\left(B_{a} K_{a} H_{b}+B_{b} K_{b} H_{a}\right) \psi \sinh (\psi / 2)\right\} .
\end{aligned}
$$

The expressions of $\mathcal{B}_{b b}, \mathcal{B}_{b a}$, and $\widehat{Q}_{b}$ are, respectively, obtained from those of $\mathcal{A}_{a a}, \mathcal{A}_{a b}$, and $\widehat{Q}_{a}$ by exchanging the species $a$ and $b$.

${ }^{1}$ M. Kogan, Rarefied Gas Dynamics (Plenum Press, New York, 1969).

${ }^{2}$ G. Em Karniadakis and A. Beskok, Micro Flows: Fundamentals and Simulation (Springer-Verlag, Berlin, 2002).

${ }^{3}$ O. Aktas, N. R. Aluru, and U. Ravaioli, "Application of a parallel DSMC technique to predict flow characteristics in microfluidic filters," J. Microelectromech. Syst. 10, 538-549 (2001).

${ }^{4}$ S. Kandlikar, S. Garimella, D. Li, S. Colin, and M. R. King, Heat Transfer and Fluid Flow in Minichannels and Microchannels (Elsevier, Paris, 2006).

${ }^{5}$ D. Lockerby and J. Reese, "High-resolution Burnett simulations of micro Couette flow and heat transfer," J. Comput. Phys. 188, 333-347 (2003).

${ }^{6}$ P. Perrier, I. Graur, T. Ewart, and J. Méolans, "Mass flow rate measurements in microtubes: From hydrodynamics to near free molecular regime," Phys. Fluids 23, 042004-042012 (2011).

${ }^{7}$ P. Bhatnagar, E. Gross, and M. Krook, "A model for collision processes in gases,” Phys. Rev. 94, 511-525 (1954).

${ }^{8}$ E. Shakhov, "Generalization of Krook kinetic relaxation equation," Fluid Dyn. 3, 95-96 (1968).

${ }^{9}$ T. Ohwada, Y. Sone, and K. Aoki, "Numerical analysis of the Poiseuille and thermal transpiration flows between two parallel plates on the basis of the linearized Boltzmann equation for hard-sphere molecules," Phys. Fluids A: Fluid Dyn. 1, 2042-2049 (1989).

${ }^{10}$ F. Sharipov, "Rarefied gas flow through a long rectangular channel," J. Vac. Sci. Technol., A 17, 3062-3066 (1999).

${ }^{11}$ F. Sharipov, "Non-isothermal gas flow through rectangular microchannels," J. Micromech. Microeng. 9, 394-401 (1999).

${ }^{12} \mathrm{~V}$. Titarev, "Implicit high-order method for calculating rarefied gas flow in a planar microchannel," J. Comput. Phys. 231, 109-134 (2012).

${ }^{13}$ G. Bird, Molecular Gas Dynamics (Clarendon Press, Oxford, 1976).

${ }^{14}$ D. Ameur, C. Croizet, F. Maroteaux, and R. Gatignol, "Modélisation des écoulements dans des micro-filtres par la méthode DSMC," La Houille Blanche, 45-50 (2007).

${ }^{15}$ F. Verhaeghe, L.-S. Luo, and B. Blanpain, "Lattice boltzmann modeling of microchannel flow in slip flow regime," J. Comput. Phys. 228, 147-157 (2009).

${ }^{16}$ T. Lee and C.-L. Lin, "Rarefaction and compressibility effects of the latticeBoltzmann-equation method in a gas microchannel," Phys. Rev. E 71, 046706-046710 (2009).

${ }^{17}$ S. Gokaltun, P. Skudarnov, M. Sukop, and G. Dulikravich, "Statistical modeling of rarefied gas flows in microchannels," in 18th AIAA Computational 
Fluid Dynamics Conference, 25-28 June 2007 (AIAA, Miami, 2007), pp. $1-14$.

${ }^{18}$ J. Shim and R. Gatignol, "Robust thermal boundary condition using Maxwell-Boltzmann statistics and its application," AIP Conf. Proc. 1333, 980-985 (2010).

${ }^{19}$ Q. Li, Y. He, G. Tang, and W. Tan, "Lattice Boltzmann modeling of microchannel flows in the transition flow regime," Microfluid. Nanofluid. 10, 607-618 (2011).

${ }^{20} \mathrm{~T}$. Reis and P. Dellar, "Lattice Boltzmann simulations of pressure-driven flows in microchannels using Navier-Maxwell slip boundary conditions," Phys. Fluids 24, 112001-112019 (2012).

${ }^{21}$ E. Arkilic, K. Breuer, and M. Schmidt, "Mass flow and tangential momentum accommodation in silicon micromachined channels," J. Fluid Mech. 437, 29-43 (2001).

${ }^{22}$ M. Fichman and G. Hetsroni, "Viscosity and slip velocity in gas flow in microchannels," Phys. Fluids 17, 123102-123105 (2005).

${ }^{23}$ N. Dongari, A. Sharma, and F. Durst, "Pressure-driven diffusive gas flows in micro-channels: From the Knudsen to the continuum regimes," Microfluid. Nanofluid. 6, 679-692 (2009).

${ }^{24}$ X. Zhu, Q. Liao, and M. D. Xin, "Gas flow in microchannel of arbitrary shape in slip flow regime," Nanoscale Microscale Thermophys. Eng. 10, 41-54 (2006).

${ }^{25}$ L. Kuddusi and E. Çetegen, "Thermal and hydrodynamic analysis of gaseous flow in trapezoidal silicon microchannels," Int. J. Therm. Sci. 48, 353-362 (2009).

${ }^{26}$ E. Arkilic, M. Schmidt, and K. Breuer, "Gaseous slip flow in long microchannels," J. Microelectromech. Syst. 6, 167-178 (1997).

${ }^{27} \mathrm{~K}$. Khasawneb, H. Liu, and C. Cai, "Gaseous slip flow in three-dimensional uniform rectangular microchannel," AIP Conf. Proc. 1333, 742-747 (2010).

${ }^{28} \mathrm{C}$. Huang, J. Gregory, and J. Sullivan, "Microchannel pressure measurements using molecular sensors," J. Microelectromech. Syst. 16, 777-785 (2007).

${ }^{29}$ S. Colin, P. Lalonde, and R. Caen, "Validation of a second-order slip flow model in rectangular microchannels," Heat Transfer Eng. 25, 23-30 (2004).

${ }^{30}$ G. Celata, M. Cumo, S. McPhail, L. Tesfagabir, and G. Zummo, "Experimental study on compressible flow in microtubes," Int. J. Heat Fluid Flow 28, 28-36 (2007).

${ }^{31}$ I. Papautsky, T. Ameel, and B. Frazier, "A review of laminar singlephase flow in microchannels," in Proceedings of 2001 ASME International Mechanical Engineering Congress and Exposition, November 11-16, 2001 (ASME, New York, 2001), pp. 1-9.

${ }^{32} \mathrm{H}$. Weng and C.-K. Chen, "On the importance of thermal creep in natural convective gas microflow with wall heat fluxes," J. Phys. D: Appl. Phys. 41, 115501-115510 (2008).

${ }^{33}$ H. Xue, H. Ji, and C. Shu, "Analysis of micro-Couette flow using Burnett equations,” Int. J. Heat Fluid Flow 44, 4139-41465 (2001).

${ }^{34}$ C. Cai, Q. Sun, and I. D. Boyd, "Gas flows in microchannels and microtubes," J. Fluid Mech. 589, 305-314 (2007).

${ }^{35}$ P. Taheri, M. Torrilhon, and H. Struchtrup, "Couette and Poiseuille microflows: Analytical solutions for regularized 13-moment equations," Phys. Fluids 21, 017102-017111 (2009).

${ }^{36}$ I. Ivchenko, S. Loyalka, and R. Tompson, "Boundary slip phenomena in a binary gas mixture," Z. Angew. Math. Phys. 53, 58-72 (2002).

${ }^{37} \mathrm{~F}$. Sharipov, "Data on the velocity slip and temperature jump on a gas-solid interface,” J. Phys. Chem. Ref. Data 40, 023101-023128 (2011).
${ }^{38}$ S. Chapman and T. Cowling, The Mathematical Theory of Non-Uniform Gases (Cambridge University Press, Cambridge, 1960).

${ }^{39} \mathrm{~F}$. McCormack, "Construction of linearized kinetic models for gaseous mixtures and molecular gases," Phys. Fluids 16, 2095-2105 (1973).

${ }^{40}$ L. Szalmás and D. Valougeorgis, "Rarefied gas flow of binary mixtures through long channels with triangular and trapezoidal cross sections," Microfluid. Nanofluid. 9, 471-487 (2010).

${ }^{41}$ L. Szalmás, "Variance-reduced DSMC for binary gas flows as defined by the McCormack kinetic model," J. Comput. Phys. 231, 3723-3738 (2012).

${ }^{42}$ L. Szalmás, "Flows of rarefied gaseous mixtures in networks of long channels," Microfluid. Nanofluid. 15, 817-827 (2013).

${ }^{43}$ L. Szalmás, J. Pitakarnnop, S. Geoffroy, S. Colin, and D. Valougeorgis, "Comparative study between computational and experimental results for binary rarefied gas flows through long microchannels," Microfluid. Nanofluid. 9, 1103-1114 (2010).

${ }^{44}$ S. Takata, S. Yasuda, S. Kosuge, and K. Aoki, "Numerical analysis of thermal-slip and diffusion-slip flows of a binary mixture of hard-sphere molecular gases," Phys. Fluids 15, 3745-3766 (2003).

${ }^{45} \mathrm{G}$. Bird, DSMC of Graeme Bird, http://gab.com.au.

${ }^{46}$ M. Reyhanian-Mashhadi, C. Croizet, and R. Gatignol, "Numerical analysis of the mixing of two gases," Mech. Ind. 14, 453-460 (2013).

${ }^{47}$ V. Gupta, H. Struchtrup, and M. Torrilhon, "Regularized moment equations for binary gas mixtures: Derivation and linear analysis," Phys. Fluids 28, 042003-042037 (2016).

${ }^{48}$ B. Rahimi and H. Struchtrup, "Macroscopic and kinetic modelling of rarefied polyatomic gases," J. Fluid Mech. 806, 437-505 (2016).

${ }^{49}$ L. Sirovich, "Kinetic modeling of gas mixtures," Phys. Fluids 5, 908-918 (1962).

${ }^{50}$ T. F. Morse, "Kinetic model equations for a gas mixture," Phys. Fluids 7, 2012-2013 (1964)

${ }^{51}$ Y. Wu and C. Lee, "Kinetic theory of shock tube problems for binary mixtures," Phys. Fluids 14, 313-322 (1971).

${ }^{52}$ T. Elizarova, I. Graur, and J.-C. Lengrand, "Two-fluid computational model for a binary gas mixture," Eur. J. Mech. B/Fluids 20, 351-369 (2001).

${ }^{53}$ M. V. Dyke, Perturbation Methods in Fluid Dynamics (Academic Press, New York, 1964).

${ }^{54}$ G. Bird, Molecular Gas Dynamics and the Direct Simulation of Gas Flows (Oxford University Press, Oxford, 1994).

${ }^{55}$ M. Reyhanian-Mashhadi, Simulation Numérique Par la Méthode de Monte Carlo (DSMC) et Modélisation Analytique d'un Mélange Gazeux Dans un Micro Canal (Thèse de l'Université Pierre et Marie Curie, Paris, 2011).

${ }^{56} \mathrm{R}$. Gatignol and C. Croizet, "Asymptotic modelling of the flow of a thermal binary mixture in a micro-channel," AIP Conf. Proc. 1628, 807-814 (2014).

${ }^{57}$ C. Croizet and R. Gatignol, "Asymptotic modelling of the axisymmetric flow of a binary gas mixture in a circular microchannel," AIP Conf. Proc.1628, 799-806 (2014)

${ }^{58}$ Y. Sone, Kinetic Theory and Fluid Dynamics (Oxford, Boston, 2002).

${ }^{59} \mathrm{R}$. Gatignol and C. Croizet, "Asymptotic modelling of the flows in microchannel by using macroscopic balance equations," AIP Conf. Proc. 1333, 730-735 (2010).

${ }^{60}$ N. Hadjiconstantinou, A. Garcia, M. Bazant, and G. He, "Statistical error in particle simulations of hydrodynamic phenomena," J. Comput. Phys. 187, 274-297 (2003).

${ }^{61}$ L. Landau and E. Lifchitz, Physique Théorique, Tome V: Physique Statistique (MIR, Moscou, 1967). 